\title{
拟线性抛物方程组具有界面外推的 并行本性差分方法
}

\section{袁光伟* 杭旭登 盛志强}

(北京应用物理与计算数学研究所, 北京 100088)

\begin{abstract}
摘要构造了拟线性抛物型方程组初边值问题的一类具有界面外推的并行本性差分格式. 为给出子区域间界面上的值或者与界面相邻点处的值, 给出了两类时间外推的方式, 得到了 二阶精度无条件稳定的并行差分格式. 并且不作启示性假定, 证明了所构造的并行差分格式 的离散向量解的存在性和唯一性. 而且在格式的离散向量解对原始问题的已知离散数据连续 依赖的意义下, 证明了并行差分格式的解按离散 $W_{2}^{(2,1)}\left(Q_{\Delta}\right)$ 范数是无条件稳定的. 最后证明 了具有界面外推的并行本性差分格式的离散向量解收敛到原始拟线性抛物问题的唯一广义 解. 给出了数值例子, 数值结果表明所构造的格式是无条件稳定的, 具有二阶精度, 且具有高 度并行性.
\end{abstract}

关键词 并行差分格式 界面外推 拟线性抛物组 无条件稳定性 收敛性 $\mathrm{MSC}(2000)$ 主题分类 $65 \mathrm{M} 06,65 \mathrm{M} 12,65 \mathrm{M} 55$

\section{1 引言}

文献 [1-5] 构造了多类求解抛物型方程的无条件稳定的并行差分格式. 文献 [1] 利用 Saul'yev 格式构造了求解扩散方程的交替分组显式 (AGE) 格式. 文献 [2] 构造了交替分段显 隐 (ASE-I) 格式, 文献 [3] 构造了纯交替分段显隐 (PASE-I) 格式. 文献 [1-3] 中格式的构造 是在时间方向上采用显隐交替技术, 这不仅导致在实际应用中存在一定的实施难度、引起新 的计算复杂性, 而且由于对一般的非线性抛物型方程组的交替格式尚未有比较深入的理论分 析, 故其稳定性和收玫阶缺乏理论保证. 文献 [4] 针对线性和非线性抛物组提出了修改的一 般交替差分格式, 并证明了这些格式的无条件稳定性. 交替分组显式格式、交替分段显隐格 式和交替分段 Crank-Nicolson 格式等均为修改的一般交替差分格式的特例, 并且可在一般格 式中特别选取参数, 得到一些新的无条件稳定的并行格式. 特别地, 在每一时间层的各个子区 域内不需采用显式格式计算, 但要求子区域的内界面在奇偶时间层进行交替移动, 使其不重 合. 所提到的这些格式实质上都是三层格式, 在应用于并行求解多维问题时不易于实际实施. 文献 [5] 所构造的并行格式是在子区域内部使用隐式计算, 在内界面处使用 Du Fort-Frankel 格式, 这也是一个三层显式计算格式.

收稿日期: 2005-07-28; 接受日期: 2006-11-09

国家重点基础研究发展计划 (批准号: 2005CB321703), 国家自然科学基金 (批准号: 10476002, 60533020) 和中国工程物理研究院科学基金 (批准号: 20060649) 资助项目

*E-mail: yuan_guangwei@iapcm.ac.cn 
无条件稳定或条件稳定的两层并行差分格式也已得到深入的研究. 文献 [6] 提出了一个 并行差分格式, 它在内界面上采用大步长的显式格式计算, 而在子区域内部采用全隐格式计 算. 文献 [7] 和 [8] 中还有一些其他的使用区域分解的格式. 但以上这 3 种格式都是条件稳定 的. 文献 [9] 对抛物问题构造了一些特殊的并行差分格式. 将整个空间区域进行区域分解后, 所构造的并行差分格式均在子区域内界面附近采用选取前一时间层的值的方法, 以实现分区 并行计算. 文献 [9] 证明了这些并行格式在离散 $L^{\infty}$ 和 $H^{1}$ 范数下是无条件稳定和收玫的, 并 且, 虽然在子区域内界面附近这些格式的截断误差为 $O(1)$, 仍证明了差分解的收玫阶为一阶, 即 $O(\tau+h)$. 采用在子区域内界面附近取前一时间层的值的方法已推广到了拟线性抛物问题 和半线性抛物问题, 并证明这些并行格式是无条件稳定和收玫的, 但其收玫阶仅仅为一阶. 为 避免在界面附近出现的精度损失, 文献 [10] 构造了基于界面修正的迭代并行格式, 其主要想 法是在子区域内部 (非线性) 迭代求解过程中进行界面隐式修正, 以保证得到无条件稳定性 和二阶精度. 总之, 以上这些并行格式都未同时满足以下 3 个要求: (i) 无条件稳定; (ii) 二阶 收敛; (iii) 实施简单.

本文将构造一些并行格式完全满足文献 [10] 中提出的 6 个要求: (i) 无条件稳定性, (ii) 二阶收玫性, (iii) 高并行度, (iv) 适应于将已有的串行程序并行化, (v) 格式设计简单, 易于实 施, (vi) 子区域上形成的代数方程组条件数较低, 可以使用已有的高效的代数方程组解法器.

为实现同时得到无条件稳定性和二阶精度这一目标, 这里引入一个新的基于时间外推的 方法. 为了同时求解子区域上的问题, 我们在子区域内界面附近取前两个时间层的值的线性 组合, 将其作为实现各子区域中的并行计算所需的内边界条件. 这种方法能保证差分格式在 界面附近的截断误差仍为 $O\left(\tau+h^{2}\right)$, 这与子区域内部隐式格式的截断误差是一致的. 于是, 在 内界面附近的差分解的精度得到提高, 从而得到整个空间区域上差分解的收玫阶为 $O\left(\tau+h^{2}\right)$.

这里构造的具有界面外推的一般并行差分格式包括两个具体的新的并行差分格式, 其中 一个在内界面处采用了 Jacobi 型的显式格式, 来得到子区域内界面处的值, 而在子区域内部 采用全隐格式. 其中在 Jacobi 型的显式格式中, 与界面相邻的点上的值取为前两个时间层的 线性组合. 另一个并行差分格式的主要构造过程如下: 首先, 在子区域间的界面上取前两个 时间层的线性组合作为子区域问题的边界条件. 然后, 在子区域内部采用全隐格式计算. 最 后, 采用全隐格式计算界面上的值, 由于与界面相邻的点上的值在前两步已经计算出来, 从而 最后一步的计算事实上是显式的.

\section{2 具有界面外推的并行差分格式}

2.1 考虑如下拟线性抛物组的初边值问题

$$
\begin{aligned}
& u_{t}=A(x, t, u) u_{x x}+f\left(x, t, u, u_{x}\right), \quad 0<x<l, \quad 0<t \leqslant T, \\
& u(0, t)=u(l, t)=0, \quad 0<t \leqslant T, \\
& u(x, 0)=\varphi(x), \quad 0 \leqslant x \leqslant l,
\end{aligned}
$$

其中 $u(x, t)=\left(u_{1}(x, t), \ldots, u_{m}(x, t)\right)$ 为 $m(m \geqslant 1)$ 维未知向量函数, $u_{t}=\frac{\partial u}{\partial t}, u_{x}=\frac{\partial u}{\partial x}$ 和 $u_{x x}=\frac{\partial^{2} u}{\partial x^{2}}$ 为对应的向量导数. $A(x, t, u)$ 是 $m \times m$ 正定系数矩阵, $f(x, t, u, p)$ 和 $\varphi(x)$ 是 $m$ 维向量函数. 记 $Q_{T}=\{0 \leqslant x \leqslant l, 0 \leqslant t \leqslant T\}$, 其中 $l>0, T>0$.

假设如下条件成立: 
(I) $A(x, t, u)$ 对 $(x, t) \in Q_{T}$ 和 $u \in \mathbb{R}^{m}$ 连续, 对 $u \in \mathbb{R}^{m}$ 一致 Lipschitz 连续. 存在常数 $\sigma_{0}>0$, 使得对任意 $\xi \in \mathbb{R}^{m}$, 所有 $(x, t) \in Q_{T}$ 和 $u \in \mathbb{R}^{m}$, 有

$$
(\xi, A(x, t, u) \xi) \geqslant \sigma_{0}|\xi|^{2} .
$$

(II) $f(x, t, u, p)$ 对 $(x, t) \in Q_{T}$ 和 $u, p \in \mathbb{R}^{m}$ 连续, 对 $u, p \in \mathbb{R}^{m}$ 一致 Lipschitz 连续. 于是, 存在常数 $C>0$, 使得 $|f(x, t, u, p)| \leqslant|\bar{f}(x, t)|+C(|u|+|p|)$ 对任意 $(x, t) \in Q_{T}$ 和 $u, p \in \mathbb{R}^{m}$ 成立, 其中 $\bar{f}(x, t)=f(x, t, 0,0)$.

(III) $\varphi(x) \in H^{1}[0, l]$ 为 $m$ 维向量函数, 满足 $\varphi(0)=\varphi(l)=0$.

2.2 用平行线 $x=x_{j}(j=0,1, \ldots, J)$ 和 $t=t^{n}(n=0,1, \ldots, N)$ 剖分 $Q_{T}$, 其中 $x_{j}=j h$, $t^{n}=n \tau$, 且 $J h=l, N \tau=T, J$ 和 $N$ 是正整数, $h$ 和 $\tau$ 为网格步长. 记 $Q_{j}^{n}=\left\{x_{j}<x \leqslant\right.$ $\left.x_{j+1}, t^{n}<t \leqslant t^{n+1}\right\}$, 其中 $j=0,1, \ldots, J-1 ; n=0,1, \ldots, N-1$. 记 $v_{\Delta}=v_{h}^{\tau}=\left\{v_{j}^{n} \mid j=\right.$ $0,1, \ldots, J ; n=0,1, \ldots, N\}$ 为定义在离散区域 $Q_{\Delta}=\left\{\left(x_{j}, t^{n}\right) \mid j=0,1, \ldots, J ; n=0,1, \ldots, N\right\}$ 上的 $m$ 维离散向量函数.

记 $r=\frac{\tau}{h^{2}}$. 记 $\Delta_{\tau} v_{j}^{n+1}=\frac{v_{j}^{n+1}-v_{j}^{n}}{\tau}, \delta v_{j+\frac{1}{2}}^{n}=\frac{v_{j+1}^{n}-v_{j}^{n}}{h}$, 且对 $n \geqslant 1$,

$$
\stackrel{*}{\delta}^{2} v_{j}^{n+1}=\frac{1}{h^{2}}\left(v_{j+1}^{\bar{n}+\lambda_{j}}-2 v_{j}^{n+1}+v_{j-1}^{\bar{n}+\mu_{j}}\right),
$$

其中 $v_{j+1}^{\bar{n}+\lambda_{j}}=\lambda_{j} v_{j+1}^{n+1}+\left(1-\lambda_{j}\right)\left(2 v_{j+1}^{n}-v_{j+1}^{n-1}\right), v_{j-1}^{\bar{n}+\mu_{j}}=\mu_{j} v_{j-1}^{n+1}+\left(1-\mu_{j}\right)\left(2 v_{j-1}^{n}-v_{j-1}^{n-1}\right)$. 当 $n=0$ 时, 定义

$$
\stackrel{*}{\delta}^{2} v_{j}^{n+1}=\delta^{2} v_{j}^{1}=\frac{1}{h^{2}}\left(v_{j+1}^{1}-2 v_{j}^{1}+v_{j-1}^{1}\right) .
$$

对拟线性抛物组初边值问题 (2.1)-(2.3) 构造如下具有界面外推的一般并行差分格式:

$$
\begin{aligned}
& \frac{v_{j}^{n+1}-v_{j}^{n}}{\tau}=A_{j}^{n+1} \stackrel{*}{\delta}^{2} v_{j}^{n+1}+f_{j}^{n+1}, \quad j=1,2, \ldots, J-1 ; n=0,1, \ldots, N-1, \\
& v_{0}^{n+1}=v_{J}^{n+1}=0, \quad n=0,1, \ldots, N-1, \\
& v_{j}^{0}=\varphi_{j}, \quad j=0,1, \ldots, J,
\end{aligned}
$$

其中 $\varphi_{j}=\varphi\left(x_{j}\right)(j=0,1, \ldots, J), \varphi_{0}=\varphi_{J}=0 ; A_{j}^{n+1}=A\left(x_{j}, t^{n+1}, \bar{\delta}^{0} v_{j}^{n+1}\right), f_{j}^{n+1}=$ $f\left(x_{j}, t^{n+1}, \hat{\delta}^{0} v_{j}^{n+1}, \bar{\delta}^{1} v_{j}^{n+1}\right)$.

对 $n \geqslant 1$, 定义差分逼近

$$
\left\{\begin{aligned}
\bar{\delta}^{0} v_{j}^{n+1}= & \lambda_{j} \alpha_{1 j}^{n} v_{j+1}^{n+1}+\alpha_{2 j}^{n} v_{j}^{n+1}+\mu_{j} \alpha_{3 j}^{n} v_{j-1}^{n+1}+\alpha_{4 j}^{n} v_{j+1}^{n}+\alpha_{5 j}^{n} v_{j}^{n}+\alpha_{6 j}^{n} v_{j-1}^{n} \\
& +\alpha_{7 j}^{n} v_{j+1}^{n-1}+\alpha_{8 j}^{n} v_{j}^{n-1}+\alpha_{9 j}^{n} v_{j-1}^{n-1}, \\
\hat{\delta}^{0} v_{j}^{n+1}= & \lambda_{j} \beta_{1 j}^{n} v_{j+1}^{n+1}+\beta_{2 j}^{n} v_{j}^{n+1}+\mu_{j} \beta_{3 j}^{n} v_{j-1}^{n+1}+\beta_{4 j}^{n} v_{j+1}^{n}+\beta_{5 j}^{n} v_{j}^{n}+\beta_{6 j}^{n} v_{j-1}^{n} \\
& +\beta_{7 j}^{n} v_{j+1}^{n-1}+\beta_{8 j}^{n} v_{j}^{n-1}+\beta_{9 j}^{n} v_{j-1}^{n-1} \\
\bar{\delta}^{1} v_{j}^{n+1}= & \gamma_{1 j}^{n} \frac{v_{j+1}^{n+\lambda_{j}}-v_{j}^{n+1}}{h}+\gamma_{2 j}^{n} \frac{v_{j}^{n+1}-v_{j-1}^{n+\mu_{j}}}{h} \\
& +\gamma_{3 j}^{n} \delta v_{j+\frac{1}{2}}^{n}+\gamma_{4 j}^{n} \delta v_{j-\frac{1}{2}}^{n}+\gamma_{5 j}^{n} \delta v_{j+\frac{1}{2}}^{n-1}+\gamma_{6 j}^{n} \delta v_{j-\frac{1}{2}}^{n-1} .
\end{aligned}\right.
$$

对 $n=0$, 定义 $A_{j}^{1}=A\left(x_{j}, t^{1}, \bar{\delta}^{0} v_{j}^{1}\right), f_{j}^{1}=f\left(x_{j}, t^{1}, \hat{\delta}^{0} v_{j}^{1}, \bar{\delta}^{1} v_{j}^{1}\right)$, 其中

$$
\begin{aligned}
& \bar{\delta}^{0} v_{j}^{1}=\alpha_{1 j}^{0} v_{j+1}^{1}+\alpha_{2 j}^{0} v_{j}^{1}+\alpha_{3 j}^{0} v_{j-1}^{1}+\alpha_{4 j}^{0} v_{j+1}^{0}+\alpha_{5 j}^{0} v_{j}^{0}+\alpha_{6 j}^{0} v_{j-1}^{0}, \\
& \hat{\delta}^{0} v_{j}^{1}=\beta_{1 j}^{0} v_{j+1}^{1}+\beta_{2 j}^{0} v_{j}^{1}+\beta_{3 j}^{0} v_{j-1}^{1}+\beta_{4 j}^{0} v_{j+1}^{0}+\beta_{5 j}^{n} v_{j}^{0}+\beta_{6 j}^{n} v_{j-1}^{0}, \\
& \bar{\delta}^{1} v_{j}^{1}=\gamma_{1 j}^{0} \delta v_{j+\frac{1}{2}}^{1}+\gamma_{2 j}^{0} \delta v_{j-\frac{1}{2}}^{1}+\gamma_{3 j}^{0} \delta v_{j+\frac{1}{2}}^{0}+\gamma_{4 j}^{0} \delta v_{j-\frac{1}{2}}^{n} .
\end{aligned}
$$




\section{3 假定}

(IV) 设 $r=\frac{\tau}{h^{2}} \leqslant \Lambda$ 对所有充分小的 $\tau$ 和 $h$ 成立, 其中 $\Lambda$ 为任一固定正常数; 对所有 $1 \leqslant j \leqslant J-1$, 有 $0 \leqslant \lambda_{j}, \mu_{j} \leqslant 1, \lambda_{j}+\mu_{j+1} \geqslant 1$, 且 (2.7) 式中的系数满足

$$
\begin{aligned}
& \lambda_{j} \alpha_{1 j}^{n}+\alpha_{2 j}^{n}+\mu_{j} \alpha_{3 j}^{n}+\alpha_{4 j}^{n}+\alpha_{5 j}^{n}+\alpha_{6 j}^{n}+\alpha_{7 j}^{n}+\alpha_{8 j}^{n}+\alpha_{9 j}^{n}=1, \\
& \lambda_{j} \beta_{1 j}^{n}+\beta_{2 j}^{n}+\mu_{j} \beta_{3 j}^{n}+\beta_{4 j}^{n}+\beta_{5 j}^{n}+\beta_{6 j}^{n}+\beta_{7 j}^{n}+\beta_{8 j}^{n}+\beta_{9 j}^{n}=1, \\
& \gamma_{1 j}^{n}+\gamma_{2 j}^{n}+\gamma_{3 j}^{n}+\gamma_{4 j}^{n}+\gamma_{5 j}^{n}+\gamma_{6 j}^{n}=1, \\
& \left|\lambda_{j} \alpha_{1 j}^{n}\right|+\left|\alpha_{2 j}^{n}\right|+\left|\mu_{j} \alpha_{3 j}^{n}\right|+\left|\alpha_{4 j}^{n}\right|+\left|\alpha_{5 j}^{n}\right|+\left|\alpha_{6 j}^{n}\right|+\left|\alpha_{7 j}^{n}\right|+\left|\alpha_{8 j}^{n}\right|+\left|\alpha_{9 j}^{n}\right| \leqslant C, \\
& \left|\lambda_{j} \beta_{1 j}^{n}\right|+\left|\beta_{2 j}^{n}\right|+\left|\mu_{j} \beta_{3 j}^{n}\right|+\left|\beta_{4 j}^{n}\right|+\left|\beta_{5 j}^{n}\right|+\left|\beta_{6 j}^{n}\right|+\left|\beta_{7 j}^{n}\right|+\left|\beta_{8 j}^{n}\right|+\left|\beta_{9 j}^{n}\right| \leqslant C, \\
& \left|\gamma_{1 j}^{n}\right|+\left|\gamma_{2 j}^{n}\right|+\left|\gamma_{3 j}^{n}\right|+\left|\gamma_{4 j}^{n}\right|+\left|\gamma_{5 j}^{n}\right|+\left|\gamma_{6 j}^{n}\right| \leqslant C .
\end{aligned}
$$

对 $n=0$, 假定类似的条件成立.

注 常数 $\alpha_{j}^{n}, \beta_{j}^{n}$ 和 $\gamma_{j}^{n}$ 依赖指标 $j=1,2, \ldots, J-1$ 和 $n=0,1, \ldots, N-1 . \lambda_{j}$ 和 $\mu_{j}$ 依 赖指标 $j=1,2, \ldots, J-1$. 对不同的 $j$ 和 $n$, 它们可以是不同的, 因此对应的差分格式有较大 的自由度. 这些格式中包含了许多具有内在并行特征的差分格式, 例如, 当在某些网格点处取 $\lambda_{j}=0$ 或 $\mu_{j}=0$ 时所得到的格式.

2.4 设 $1<k<J-1$. 当 $\lambda_{k}=0$, 易见在 $x_{k}$ 左边的子区域 $\left\{x_{j} \mid j \leqslant k\right\}$ 上的 $\left\{v_{j}^{n+1} \mid j \leqslant k\right\}$ 与右 边子区域 $\left\{x_{j} \mid j>k\right\}$ 上的 $\left\{v_{j}^{n+1} \mid j>k\right\}$ 不耦合. 同样, 当 $\mu_{k}=0$, 在 $x_{k}$ 右边子区域 $\left\{x_{j} \mid j \geqslant k\right\}$ 上的 $\left\{v_{j}^{n+1} \mid j \geqslant k\right\}$ 与左边子区域上的 $\left\{v_{j}^{n+1} \mid j<k\right\}$ 不耦合. 于是, 只要分别适当选取某些 $\lambda_{j}$ 和 $\mu_{j}$ 为 0 , 则可以自然地实现并行计算. 例如, 若对某个 $2<k<J-2$, 有 $\lambda_{k}=\mu_{k+2}=0$, 其 余 $\lambda_{j}$ 和 $\mu_{j}$ 为 1 , 即可得到文献 [8] 中的并行格式 (S1) 的修正格式. 即在 $j=k+1$ 处取内边 界条件 $2 v_{k+1}^{n}-v_{k+1}^{n-1}$, 分别在子区域 $\{j<k+1\}$ 和 $\{j>k+1\}$ 上采用隐式格式进行并行计 算, 然后在 $j=k+1$ 采用隐式格式进行计算 (这时利用已经计算出的 $v_{k}^{n+1}$ 和 $v_{k+2}^{n+1}$ ).

为进一步理解格式 (2.4)-(2.6) 的构造, 特别地考察求解 $u_{t}=u_{x x}$ 的并行格式. 首先使用 如下格式并行求解子区域 $\left\{x_{j} \mid j<k\right\}$ 与 $\left\{x_{j} \mid j>k\right\}$ 上的值 $\left\{v_{j}^{n+1} \mid j \neq k\right\}$ :

$$
\begin{aligned}
& \Delta_{\tau} v_{k-1}^{n+1}=\frac{1}{h^{2}}\left(\left(2 v_{k}^{n}-v_{k}^{n-1}\right)-2 v_{k-1}^{n+1}+v_{k-2}^{n+1}\right), \\
& \Delta_{\tau} v_{j}^{n+1}=\frac{1}{h^{2}}\left(v_{j+1}^{n+1}-2 v_{j}^{n+1}+v_{j}^{n+1}\right), \quad j \neq k-1, k, k+1, \\
& \Delta_{\tau} v_{k+1}^{n+1}=\frac{1}{h^{2}}\left(v_{k+2}^{n+1}-2 v_{k+1}^{n+1}+\left(2 v_{k}^{n}-v_{k}^{n-1}\right)\right),
\end{aligned}
$$

然后使用下式求出 $v_{k}^{n+1}$ :

$$
\Delta_{\tau} v_{k}^{n+1}=\frac{1}{h^{2}}\left(v_{k+1}^{n+1}-2 v_{k}^{n+1}+v_{k}^{n+1}\right) .
$$

该格式即为 $(2.4)$ 式的特例, 其中取 $\lambda_{k-1}=0, \mu_{k+1}=0$, 其余 $\lambda_{j}$ 和 $\mu_{j}$ 均为 1 . 注意到 $\Delta_{\tau} v_{k}^{n}=\delta^{2} v_{k}^{n}$. 该格式可改写为

$$
\begin{aligned}
& \Delta_{\tau} v_{k-1}^{n+1}=\frac{1}{h^{2}}\left(v_{k}^{n}-2 v_{k-1}^{n+1}+v_{k-2}^{n+1}\right)+r \delta^{2} v_{k}^{n}, \\
& \Delta_{\tau} v_{j}^{n+1}=\frac{1}{h^{2}}\left(v_{j+1}^{n+1}-2 v_{j}^{n+1}+v_{j}^{n+1}\right), j \neq k-1, k+1, \\
& \Delta_{\tau} v_{k+1}^{n+1}=\frac{1}{h^{2}}\left(v_{k+2}^{n+1}-2 v_{k+1}^{n+1}+v_{k}^{n}\right)+r \delta^{2} v_{k}^{n} .
\end{aligned}
$$

显然, 该格式实质上为两层差分格式, 且可以自然地并行求解. 这表明, 虽然 (2.4) 式在内界 
面处形式上涉及 3 个时间层的值, 但其中所包含的许多并行格式事实上是两层格式. 将在本 文第 7 节中进一步给出一些具体的并行格式的构造的例子.

2.5 对离散函数 $u_{h}=\left\{u_{j} \mid j=0,1, \ldots, J\right\}$, 其中 $u_{0}=u_{J}=0$, 定义离散范数

$$
\left\|u_{h}\right\|_{\infty}=\max _{0 \leqslant j \leqslant J}\left|u_{j}\right|, \quad\left\|u_{h}\right\|_{2}^{2}=\sum_{j=1}^{J-1}\left|u_{j}\right|^{2} h, \quad\left\|\delta u_{h}\right\|_{2}^{2}=\sum_{j=0}^{J-1}\left|\delta u_{j+\frac{1}{2}}\right|^{2} h .
$$

将需要如下 3 个引理 ${ }^{[11]}$.

引理 2.1 (离散 Green 公式) 令 $u_{j}$ 和 $v_{j}$ 为定义于 $\left\{x_{j} \mid j=0,1, \ldots, J\right\}$ 上的离散函数, 则

$$
\sum_{j=0}^{J-1} u_{j}\left(v_{j+1}-v_{j}\right)=-\sum_{j=1}^{J-1}\left(u_{j}-u_{j-1}\right) v_{j}-u_{0} v_{0}+u_{J-1} v_{J} .
$$

引理 2.2 (离散 Gronwall 不等式) (i) 令 $w^{n} \geqslant 0$ 为定义于 $\left\{t^{n} \mid n=0,1, \ldots, N\right\}$ 上的离 散函数, 且满足

$$
w^{n+1}-w^{n} \leqslant B \tau\left(w^{n+1}+w^{n}\right)+C_{n} \tau, \quad n=0,1, \ldots, N-1,
$$

其中 $B$ 和 $C_{n}$ 为非负常数, $N \tau=T$, 则

$$
w^{n} \leqslant\left(w^{0}+\sum_{k=0}^{N} C_{k} \tau\right) e^{4 B T}, \quad n=0,1, \ldots, N,
$$

其中 $\tau$ 使得 $4 B \tau \leqslant \frac{N-1}{N}$.

(ii) 假定离散函数 $w^{\tau}=\left\{w^{n} \geqslant 0 \mid n=0,1, \ldots, N\right\}, N \tau=T$, 满足不等式

$$
w^{n} \leqslant B+C \sum_{k=0}^{n} w^{k} \tau,
$$

其中 $B$ 和 $C$ 为非负常数, 则

$$
w^{n} \leqslant B\left(e^{2 C T}+1\right)
$$

其中 $\tau$ 使得 $C \tau \leqslant \frac{1}{2}$.

(iii) 令 $w^{n} \geqslant 0$ 为定义于 $\left\{t^{n} \mid n=0,1, \ldots, N\right\}$ 上的离散函数, 且满足

$$
w^{n+1}-w^{n} \leqslant B \tau\left(w^{n-1}+w^{n}+w^{n+1}\right)+C_{n} \tau, \quad n=1, \ldots, N-1,
$$

其中 $B$ 和 $C_{n}$ 为非负常数, $N \tau=T$, 则当 $n \geqslant 1$ 时,

$$
w^{n+1} \leqslant 3 B \sum_{k=0}^{n+1} w^{k} \tau+G^{n}, \quad n=1, \ldots, N-1,
$$

且

$$
w^{n+1} \leqslant\left(3 B\left(w^{0}+w^{1}\right) \tau+2 G^{n}\right) e^{6 B T}, \quad n=1, \ldots, N-1,
$$

其中 $G^{n}=w^{1}+\sum_{k=1}^{n} C_{k} \tau, \tau$ 使得 $2 B \tau \leqslant 1$.

这里仅仅给出 (iii) 的证明. 记

$$
F^{n+1}=\sum_{k=0}^{n+1} w^{k} \tau, \quad G^{n}=\sum_{k=1}^{n} C_{k} \tau+w^{1},
$$

则有 $w^{n+1} \leqslant 3 B F^{n+1}+G^{n}$. 于是,

$$
F^{n+1} \leqslant \frac{1}{1-3 B \tau} F^{n}+\frac{\tau}{1-3 B \tau} G^{n}
$$




$$
\begin{aligned}
& \leqslant \frac{1}{(1-3 B \tau)^{n}} F^{1}+\frac{\tau}{(1-3 B \tau)^{n-1}} G^{2}+\ldots+\frac{\tau}{1-3 B \tau} G^{n} \\
& \leqslant \frac{1}{(1-3 B \tau)^{n}}\left(w^{1}+w^{0}\right) \tau+\frac{1}{3 B}\left(\frac{\tau}{(1-3 B \tau)^{n-1}}-1\right) G^{n} \\
& \leqslant\left(\left(w^{0}+w^{1}\right) \tau+\frac{G^{n}}{3 B}\right) e^{6 B T} .
\end{aligned}
$$

所以, (iii) 获证.

引理 $\mathbf{2 . 3}$ (内插公式) 对任意离散函数 $u_{h}=\left\{u_{j} \mid j=0,1, \ldots, J\right\}(J h=l)$, 有

(i) 任给 $\varepsilon>0$, 成立

$$
\left\|u_{h}\right\|_{\infty}^{2} \leqslant \varepsilon\left\|\delta u_{h}\right\|_{2}^{2}+\frac{C}{\varepsilon}\left\|u_{h}\right\|_{2}^{2},
$$

其中 $C$ 为与 $l$ 有关的常数, 但与 $\varepsilon, h$ 和 $u_{h}$ 无关;

(ii) 如果 $u_{0}=u_{J}=0$, 则

$$
\left\|u_{h}\right\|_{2} \leqslant l\left\|\delta u_{h}\right\|_{2}, \quad\left\|u_{h}\right\|_{\infty} \leqslant\left\|\delta u_{h}\right\|_{2}^{\frac{1}{2}}\left\|u_{h}\right\|_{2}^{\frac{1}{2}} ;
$$

(iii) 存在常数 $C$ 与 $h$ 和 $l$ 无关, 使得

$$
\left\|\delta u_{h}\right\|_{2} \leqslant C\left(\left\|u_{h}\right\|_{2}^{\frac{1}{2}}\left\|\delta^{2} u_{h}\right\|_{2}^{\frac{1}{2}}+l^{-1}\left\|u_{h}\right\|_{2}\right) .
$$

在本文中 $C$ 为与 $h, \tau$ 和 $v_{h}^{\tau}$ 无关的常数, 且在不同地方可以是不同的. 记

$$
\left\|\delta^{*} v_{h}^{n+1}\right\|_{2}^{2}=\sum_{j=1}^{J-1}\left|\delta^{2} v_{j}^{n+1}\right|^{2} h, \quad\left\|\Delta_{\tau} v_{h}^{n+1}\right\|_{2}^{2}=\sum_{j=1}^{J-1}\left|\Delta_{\tau} v_{j}^{n+1}\right|^{2} h .
$$

\section{3 先验估计与存在性}

3.1 本节将证明并行格式 (2.4)-(2.6) 的离散解的存在性. 首先给出离散解的先验估计.

用向量 $\delta^{*} v_{j}^{n+1} h \tau$ 与 (2.4) 式作内积, 并对 $j=1,2, \ldots, J-1$ 求和, 得

$$
\sum_{j=1}^{J-1}\left(\stackrel{*}{\delta}^{2} v_{j}^{n+1}, v_{j}^{n+1}-v_{j}^{n}\right) h=\tau \sum_{j=1}^{J-1}\left({ }^{*} \delta^{2} v_{j}^{n+1}, A_{j}^{n+1} \stackrel{*}{\delta}^{2} v_{j}^{n+1}+f_{j}^{n+1}\right) h .
$$

显然

$$
\delta^{*}{ }^{2} v_{j}^{n+1}=\delta^{2} v_{j}^{n+1}-r\left[\left(1-\lambda_{j}\right)\left(\Delta_{\tau} v_{j+1}^{n+1}-\Delta_{\tau} v_{j+1}^{n}\right)+\left(1-\mu_{j}\right)\left(\Delta_{\tau} v_{j-1}^{n+1}-\Delta_{\tau} v_{j-1}^{n}\right)\right] .
$$

且

$$
\sum_{j=1}^{J-1}\left(\delta^{2} v_{j}^{n+1}, v_{j}^{n+1}-v_{j}^{n}\right) h=-\frac{1}{2}\left\|\delta v_{h}^{n+1}\right\|_{2}^{2}+\frac{1}{2}\left\|\delta v_{h}^{n}\right\|_{2}^{2}-\frac{1}{2}\left\|\delta v_{h}^{n+1}-\delta v_{h}^{n}\right\|_{2}^{2} .
$$

当 $0 \leqslant \lambda_{j} \leqslant 1,0 \leqslant \mu_{j} \leqslant 1, \lambda_{j}+\mu_{j+1} \geqslant 1$ 时, 有

$$
\begin{aligned}
-r \sum_{j=1}^{J-1}\left(\left(1-\lambda_{j}\right)\left(\Delta_{\tau} v_{j+1}^{n+1}-\Delta_{\tau} v_{j+1}^{n}\right)\right. & \left.+\left(1-\mu_{j}\right)\left(\Delta_{\tau} v_{j-1}^{n+1}-\Delta_{\tau} v_{j-1}^{n}\right), v_{j}^{n+1}-v_{j}^{n}\right) h \\
-\frac{1}{2}\left\|\delta v_{h}^{n+1}-\delta v_{h}^{n}\right\|_{2}^{2} \leqslant \frac{\tau^{2}}{2 h} \sum_{j=0}^{J-1}[ & -\left(1-\lambda_{j}\right)\left|\Delta_{\tau} v_{j+1}^{n+1}\right|^{2}-\left(1-\mu_{j+1}\right)\left|\Delta_{\tau} v_{j}^{n+1}\right|^{2} \\
& \left.+\left(1-\lambda_{j}\right)\left|\Delta_{\tau} v_{j+1}^{n}\right|^{2}+\left(1-\mu_{j+1}\right)\left|\Delta_{\tau} v_{j}^{n}\right|^{2}\right]
\end{aligned}
$$




$$
=-\frac{\tau r}{2} \sum_{j=1}^{J-1}\left(2-\lambda_{j-1}-\mu_{j+1}\right)\left(\left|\Delta_{\tau} v_{j}^{n+1}\right|^{2}-\left|\Delta_{\tau} v_{j}^{n}\right|^{2}\right) h .
$$

其中利用了如下初等不等式:

$$
\begin{gathered}
-\frac{1}{2}\left|a_{j+1}-a_{j}\right|^{2}-\left(2-\lambda_{j}-\mu_{j+1}\right)\left(a_{j+1}, a_{j}\right)+\left(1-\lambda_{j}\right)\left(b_{j+1}, a_{j}\right)+\left(1-\mu_{j+1}\right)\left(b_{j}, a_{j+1}\right) \\
\leqslant-\frac{1}{2}\left(1-\lambda_{j}\right)\left|a_{j+1}\right|^{2}-\frac{1}{2}\left(1-\mu_{j+1}\right)\left|a_{j}\right|^{2}+\frac{1}{2}\left(1-\lambda_{j}\right)\left|b_{j+1}\right|^{2}+\frac{1}{2}\left(1-\mu_{j+1}\right)\left|b_{j}\right|^{2},
\end{gathered}
$$

其中 $\forall a_{j}, a_{j+1}, b_{j}, b_{j+1} \in \mathbb{R}^{m}$. 于是得

$$
\begin{aligned}
& \left\|\delta v_{h}^{n+1}\right\|_{2}^{2}-\left\|\delta v_{h}^{n}\right\|_{2}^{2}+2 \tau \sum_{j=1}^{J-1}\left({ }^{*}{ }^{2} v_{j}^{n+1}, A_{j}^{n+1} \stackrel{*}{\delta}^{2} v_{j}^{n+1}\right) \\
& +\tau r \sum_{j=1}^{J-1}\left(2-\lambda_{j-1}-\mu_{j+1}\right)\left(\left|\Delta_{\tau} v_{j}^{n+1}\right|^{2}-\left|\Delta_{\tau} v_{j}^{n}\right|^{2}\right) h \\
& \leqslant-2 \tau \sum_{j=0}^{J-1}\left({ }^{*}{ }^{2} v_{j}^{n+1}, f_{j}^{n+1}\right) h \leqslant 2 \tau\left|\sum_{j=1}^{J-1}\left({ }^{*}{ }^{2} v_{j}^{n+1}, f_{j}^{n+1}\right) h\right| \\
& \leqslant \frac{\tau}{2} \sum_{j=1}^{J-1}\left({ }^{*}{ }^{2} v_{j}^{n+1}, A_{j}^{n+1}{ }^{*}{ }^{2} v_{j}^{n+1}\right) h+2 \tau \sum_{j=1}^{J-1} \frac{\left|f_{j}^{n+1}\right|^{2}}{\sigma\left(A_{j}^{n+1}\right)} h,
\end{aligned}
$$

其中对上式右端应用了 Cauchy 不等式, 且

$$
\sigma(A)=\inf _{\xi \in \mathbb{R}^{m}} \frac{(\xi, A \xi)}{|\xi|^{2}} .
$$

3.2 利用差分组 (2.4), 对任意 $\varepsilon>0$, 有

$$
\begin{aligned}
\varepsilon \sum_{j=1}^{J-1}\left|\Delta_{\tau} v_{j}^{n+1}\right|^{2} h & =\varepsilon \sum_{j=1}^{J-1}\left|A_{j}^{n+1} \stackrel{*}{\delta}^{2} v_{j}^{n+1}+f_{j}^{n+1}\right|^{2} h \\
& \leqslant 2 \varepsilon \sum_{j=1}^{J-1} \frac{\rho^{2}\left(A_{j}^{n+1}\right)}{\sigma\left(A_{j}^{n+1}\right)}\left({ }^{*}{ }^{2} v_{j}^{n+1}, A_{j}^{n+1} \stackrel{*}{\delta}^{2} v_{j}^{n+1}\right) h+2 \varepsilon \sum_{j=1}^{J-1}\left|f_{j}^{n+1}\right|^{2} h,
\end{aligned}
$$

其中

$$
\rho(A)=\sup _{\xi \in=\mathbb{R}^{m}} \frac{|A \xi|}{|\xi|} .
$$

由条件(II) 和定义 (2.7),

$$
\begin{gathered}
\sum_{j=1}^{J-1}\left|f_{j}^{n+1}\right|^{2} h \leqslant C \\
\left(1+\left\|v_{h}^{n-1}\right\|_{2}^{2}+\left\|v_{h}^{n}\right\|_{2}^{2}+\left\|v_{h}^{n+1}\right\|_{2}^{2}+\left\|\delta v_{h}^{n-1}\right\|_{2}^{2}+\left\|\delta v_{h}^{n}\right\|_{2}^{2}+\left\|\delta v_{h}^{n+1}\right\|_{2}^{2}\right. \\
\left.+\tau \Lambda\left\|\Delta_{\tau} v_{h}^{n}\right\|^{2}+\tau \Lambda\left\|\Delta_{\tau} v_{h}^{n+1}\right\|^{2}\right) .
\end{gathered}
$$

联合 (3.2)-(3.4) 式, 并取 $\varepsilon>0$, 使得

$$
\varepsilon \max _{(x, t) \in Q_{T}, u \in \mathbb{R}^{m}} \frac{\rho^{2}(A)}{\sigma(A)} \leqslant \frac{1}{4},
$$

得

$$
\left\|\delta v_{h}^{n+1}\right\|_{2}^{2}-\left\|\delta v_{h}^{n}\right\|_{2}^{2}+\tau r \sum_{j=1}^{J-1}\left(2-\lambda_{j-1}-\mu_{j+1}\right)\left(\left|\Delta_{\tau} v_{j}^{n+1}\right|^{2}-\left|\Delta_{\tau} v_{j}^{n}\right|^{2}\right) h
$$




$$
\begin{aligned}
& +2 \tau \sum_{j=1}^{J-1}\left(\stackrel{*}{\delta}^{2} v_{j}^{n+1}, A_{j}^{n+1} \stackrel{*}{\delta}^{2} v_{j}^{n+1}\right) h+\varepsilon \tau\left\|\Delta_{\tau} v_{h}^{n+1}\right\|_{2}^{2} \\
& \leqslant \tau \sum_{j=1}^{J-1}\left(\stackrel{*}{\delta}^{2} v_{j}^{n+1}, A_{j}^{n+1} \stackrel{*}{\delta}^{2} v_{j}^{n+1}\right) h+2 \tau \sum_{j=1}^{J-1}\left(\frac{1}{\sigma\left(A_{j}^{n+1}\right)}+\varepsilon\right)\left|f_{j}^{n+1}\right|^{2} h \\
& \leqslant \tau \sum_{j=1}^{J-1}\left({ }^{*}{ }^{2} v_{j}^{n+1}, A_{j}^{n+1} \stackrel{*}{\delta}^{2} v_{j}^{n+1}\right) h+C \tau\left(1+\left\|\delta v_{h}^{n-1}\right\|_{2}^{2}+\left\|\delta v_{h}^{n}\right\|_{2}^{2}\right. \\
& \left.+\left\|\delta v_{h}^{n+1}\right\|_{2}^{2}+\tau \Lambda\left\|\Delta_{\tau} v_{h}^{n}\right\|^{2}+\tau \Lambda\left\|\Delta_{\tau} v_{h}^{n+1}\right\|^{2}\right) .
\end{aligned}
$$

这里利用了条件(I) 和引理 2.3 (ii). 取 $C \tau \Lambda \leqslant \frac{\varepsilon}{2}$, 得

$$
\begin{gathered}
\left\|\delta v_{h}^{n+1}\right\|_{2}^{2}-\left\|\delta v_{h}^{n}\right\|_{2}^{2}+\tau r \sum_{j=1}^{J-1}\left(2-\lambda_{j-1}-\mu_{j+1}\right)\left(\left|\Delta_{\tau} v_{j}^{n+1}\right|^{2}-\left|\Delta_{\tau} v_{j}^{n}\right|^{2}\right) h \\
+\tau \sigma_{0} \sum_{j=1}^{J-1}\left|{ }^{*}{ }^{2} v_{j}^{n+1}\right|^{2} h+\frac{\varepsilon \tau}{2}\left(\left\|\Delta_{\tau} v_{h}^{n+1}\right\|_{2}^{2}-\left\|\Delta_{\tau} v_{h}^{n}\right\|_{2}^{2}\right) \\
\leqslant C \tau\left(1+\left\|\delta v_{h}^{n-1}\right\|_{2}^{2}+\left\|\delta v_{h}^{n}\right\|_{2}^{2}+\left\|\delta v_{h}^{n+1}\right\|_{2}^{2}\right) .
\end{gathered}
$$

3.3 对 $n \geqslant 1$,

$$
\begin{aligned}
& \left\|\delta v_{h}^{n+1}\right\|_{2}^{2}+\tau r \sum_{j=1}^{J-1}\left(2-\lambda_{j-1}-\mu_{j+1}\right)\left|\Delta_{\tau} v_{j}^{n+1}\right|^{2}+\tau \sigma_{0} \sum_{j=1}^{J-1}\left|{ }^{*}{ }^{2} v_{j}^{n+1}\right|^{2} h+\frac{\varepsilon \tau}{2}\left\|\Delta_{\tau} v_{h}^{n+1}\right\|_{2}^{2} \\
& \leqslant C\left(1+\sum_{k=0}^{n+1}\left\|\delta v_{h}^{k}\right\|_{2}^{2} \tau+r \tau\left\|\Delta_{\tau} v_{h}^{1}\right\|_{2}^{2}\right) .
\end{aligned}
$$

利用 $n=0$ 时的隐式格式, 可推出

$$
\left\|\delta v_{h}^{1}\right\|_{2}^{2}+\tau\left\|\Delta_{\tau} v_{h}^{1}\right\|_{2}^{2} \leqslant C\left(1+\left\|\delta v_{h}^{0}\right\|_{2}^{2}\right) .
$$

联合以上两式, 并利用引理 2.2 和 2.3 , 得

$$
\begin{aligned}
& \max _{1 \leqslant n \leqslant N}\left(\left\|\delta v_{h}^{n}\right\|_{2},\left\|v_{h}^{n}\right\|_{2}\right) \leqslant C\left(1+\left\|\delta \varphi_{h}\right\|_{2}\right), \\
& \left(\sum_{n=0}^{N-1}\left\|\delta^{*} v_{h}^{n+1}\right\|_{2}^{2} \tau\right)^{\frac{1}{2}}+\left(\sum_{n=0}^{N-1}\left\|\Delta_{\tau} v_{h}^{n+1}\right\|_{2}^{2} \tau\right)^{\frac{1}{2}} \leqslant C .
\end{aligned}
$$

显然

$$
\delta^{2} v_{j}^{n+1}=\stackrel{*}{\delta}^{2} v_{j}^{n+1}+r\left[\left(1-\lambda_{j}\right)\left(\Delta_{\tau} v_{j+1}^{n+1}-\Delta_{\tau} v_{j+1}^{n}\right)+\left(1-\mu_{j}\right)\left(\Delta_{\tau} v_{j-1}^{n+1}-\Delta_{\tau} v_{j-1}^{n}\right)\right] .
$$

于是, 由 (3.7) 和 (3.8) 式以及引理 2.3 , 有

$$
\left(\sum_{n=0}^{N-1}\left\|\delta^{2} v_{h}^{n+1}\right\|_{2}^{2} \tau\right)^{\frac{1}{2}}+\max _{n=0,1, \ldots, N-1}\left\|v_{h}^{n+1}\right\|_{\infty} \leqslant C .
$$

\section{4 我们证明了如下结果:}

定理 1 设条件(I)-(IV)成立, 且对所有 $0 \leqslant j \leqslant J$, 成立 $0 \leqslant \lambda_{j} \leqslant 1,0 \leqslant \mu_{j} \leqslant 1, \lambda_{j}+$ $\mu_{j+1} \geqslant 1$. 那么, 存在仅依赖已知数据和 $\Lambda$ 的正常数 $\tau_{0}$, 当 $\tau \leqslant \tau_{0}$ 时, 并行差分格式 (2.4)-(2.6) 的解满足估计 (3.7)-(3.9). 
利用有限维空间上 Leray-Schauder 不动点定理 ${ }^{[11]}$ 以及估计 (3.7)-(3.9), 可得并行格式 (2.4)-(2.6) 解 $v_{\Delta}=v_{h}^{\tau}=\left\{v_{j}^{n} \mid j=0,1, \ldots, J ; n=0,1, \ldots, N\right\}$ 的存在性.

定理 2 设定理 1 的条件成立, 则存在仅依赖已知数据和 $\Lambda$ 的正常数 $\tau_{0}$, 当 $\tau \leqslant \tau_{0}$ 时, 并行差分格式 (2.4)-(2.6) 至少存在一个解 $v_{\Delta}=v_{h}^{\tau}=\left\{v_{j}^{n} \mid j=0,1, \ldots, J ; n=0,1, \ldots, N\right\}$.

\section{4 唯一性}

4.1 给定差分格式 (2.4)-(2.6) 在第 $n-1$ 层和第 $n$ 层的值: $\left\{v_{j}^{n-1} \mid j=0,1, \ldots, J\right\},\left\{v_{j}^{n} \mid j=\right.$ $0,1, \ldots, J\}$. 令 $\left\{v_{j}^{n+1} \mid j=0,1, \ldots, J\right\}$ 和 $\left\{\bar{v}_{j}^{n+1} \mid j=0,1, \ldots, J\right\}$ 为格式 $(2.4)-(2.6)$ 在第 $(n+1)$ 层上的两个解. 当 $n=0$ 时, 显然有 $v_{j}^{1}=\bar{v}_{j}^{1}, 0 \leqslant j \leqslant J$. 下面设 $n \geqslant 1$, 则有

$$
\frac{v_{j}^{n+1}-v_{j}^{n}}{\tau}=A_{j}^{n+1} \stackrel{*}{\delta}{ }^{2} v_{j}^{n+1}+f_{j}^{n+1} \quad(j=1,2, \ldots, J-1), \quad v_{0}^{n+1}=v_{J}^{n+1}=0,
$$

和

$$
\frac{\bar{v}_{j}^{n+1}-v_{j}^{n}}{\tau}=\bar{A}_{j}^{n+1} \stackrel{\delta}{\delta}^{2} \bar{v}_{j}^{n+1}+\bar{f}_{j}^{n+1} \quad(j=1,2, \ldots, J-1), \quad \bar{v}_{0}^{n+1}=\bar{v}_{J}^{n+1}=0,
$$

其中 $\bar{A}_{j}^{n+1}$ 和 $\bar{f}_{j}^{n+1}(j=1,2, \ldots, J-1)$ 可分别由 $A_{j}^{n+1}$ 和 $f_{j}^{n+1}(j=1,2, \ldots, J-1)$ 将 $v_{j}^{n+1}$ $(j=0,1, \ldots, J)$ 用 $\bar{v}_{j}^{n+1}(j=0,1, \ldots, J)$ 替代而得到. 记 $w_{j} \equiv v_{j}^{n+1}-\bar{v}_{j}^{n+1}$, 于是有

$$
\begin{aligned}
& w_{j}=\tau A_{j}^{n+1} \stackrel{*}{\delta}^{2} w_{j}+\tau R_{j}^{n} \quad(j=1,2, \ldots, J-1), \\
& w_{0}=w_{J}=0,
\end{aligned}
$$

其中 ${ }^{*}{ }^{2} w_{j}={ }^{*}{ }^{2} v_{j}^{n+1}-{ }^{*}{ }^{2} \bar{v}_{j}^{n+1}=\frac{1}{h^{2}}\left(\lambda_{j} w_{j+1}-2 w_{j}+\mu_{j} w_{j-1}\right)$, 且

$$
R_{j}^{n}=\left(A_{j}^{n+1}-\bar{A}_{j}^{n+1}\right) \stackrel{\kappa}{\delta}^{2} \bar{v}_{j}^{n+1}+f_{j}^{n+1}-\bar{f}_{j}^{n+1} .
$$

4.2 用向量 ${ }^{*}{ }^{2} w_{j} h \tau$ 与 (4.1) 式作内积, 并对 $j=1,2, \ldots, J-1$ 求和, 然后应用与第 3 节中 类似的推导, 得

$$
\left\|\delta w_{h}\right\|_{2}^{2}+2 \tau \sum_{j=1}^{J-1}\left({ }^{*} \delta^{2} w_{j}, A_{j}^{n+1} \stackrel{*}{\delta}^{2} w_{j}\right) h+\tau \sum_{j=1}^{J-1}\left(2-\lambda_{j-1}-\mu_{j+1}\right) w_{j}^{2} h \leqslant 2 \tau \sum_{j=1}^{J-1}\left|\left(\stackrel{*}{\delta}^{2} w_{j}, R_{j}^{n}\right)\right| h .
$$

可得

$$
\left\|\delta w_{h}\right\|_{2}^{2}+\tau \sigma_{0}\left\|\delta^{*}{ }^{2} w_{h}\right\|_{2}^{2} \leqslant C \tau \sum_{j=1}^{J-1}\left|R_{j}^{n}\right|^{2} h .
$$

由条件(I) 和定义 (2.1), 并利用引理 2.3 (i), 可推出对任意 $\varepsilon_{1}>0$,

$$
\begin{aligned}
& \left\|A_{h}^{n+1}-\bar{A}_{h}^{n+1}\right\|_{\infty}^{2} \leqslant \varepsilon_{1}\left\|\delta w_{h}\right\|_{2}^{2}+\frac{C}{\varepsilon_{1}}\left\|w_{h}\right\|_{2}^{2}, \\
& \left\|f_{h}^{n+1}-\bar{f}_{h}^{n+1}\right\|_{2}^{2} \leqslant C\left(\left\|\delta w_{h}\right\|_{2}^{2}+\frac{1}{h} \sum_{j=1}^{J-1}\left(\left|\lambda_{j}\right|^{2} w_{j+1}^{2}+\left|\mu_{j}\right|^{2} w_{j-1}^{2}\right)\right) .
\end{aligned}
$$

并且, 由估计 (3.8), 有

$$
\tau\left\|\stackrel{*}{\delta} \bar{v}_{h}^{n+1}\right\|_{2}^{2} \leqslant C,
$$

因此

$$
\tau \sum_{j=1}^{J-1}\left|R_{j}^{n}\right|^{2} h \leqslant C\left(\varepsilon_{1}+\tau\right)\left\|\delta w_{h}\right\|_{2}^{2}+C \Lambda\left\|w_{h}\right\|_{2}^{2}
$$


将 (4.4) 式代入 (4.3) 式, 得

$$
\left\|\delta w_{h}\right\|_{2}^{2}+\tau \sigma_{0}\left\|\delta^{*} w_{h}\right\|_{2}^{2} \leqslant C\left(\varepsilon_{1}+\tau\right)\left\|\delta w_{h}\right\|_{2}^{2}+C \Lambda\left\|w_{h}\right\|_{2}^{2} .
$$

取 $\varepsilon_{1} \leqslant \frac{1}{4 C}$ 和 $\tau \leqslant \frac{1}{4 C}$, 得

$$
\left\|\delta w_{h}\right\|_{2}^{2}+\tau \sigma_{0}\left\|\stackrel{*}{\delta}^{2} w_{h}\right\|_{2}^{2} \leqslant C\left\|w_{h}\right\|_{2}^{2}
$$

其中 $C$ 仅依赖已知数据.

4.3 为估计 $\left\|w_{h}\right\|_{2}^{2}$, 用向量 $w_{j} h$ 与 (4.1) 式作内积, 并对 $j=1,2, \ldots, J-1$ 求和, 得到

$$
\left\|w_{h}\right\|_{2}^{2}=\tau \sum_{j=1}^{J-1}\left(w_{j}, A_{j}^{n+1} \stackrel{*}{\delta}^{2} w_{j}\right) h+\tau \sum_{j=1}^{J-1}\left(w_{j}, R_{j}^{n}\right) h \equiv I+I I .
$$

对任意 $\varepsilon_{2}>0$ 和 $\varepsilon_{3}>0$, 有

$$
\begin{aligned}
& \mathrm{I} \leqslant \tau\left(\varepsilon_{2}\left\|\delta^{*} w_{h}\right\|_{2}^{2}+\frac{C}{\varepsilon_{2}}\left\|w_{h}\right\|_{2}^{2}\right), \\
& \mathrm{II} \leqslant \frac{\tau}{\varepsilon_{3}}\left\|w_{h}\right\|_{2}^{2}+\varepsilon_{3} \tau \sum_{j=1}^{J-1}\left|R_{j}^{n}\right|^{2} h \leqslant C \varepsilon_{3}(1+\tau)\left\|\delta w_{h}\right\|_{2}^{2}+\left(\varepsilon_{3} C \Lambda+\frac{\tau}{\varepsilon_{3}}\right)\left\|w_{h}\right\|_{2}^{2} .
\end{aligned}
$$

将上面两个不等式代入 (4.6) 式, 得

$$
\left\|w_{h}\right\|_{2}^{2} \leqslant 2\left(C \varepsilon_{3} \Lambda+\frac{\tau}{\varepsilon_{3}}+\frac{C \tau}{\varepsilon_{2}}\right)\left\|w_{h}\right\|_{2}^{2}+C \varepsilon_{3}(1+\tau)\left\|\delta w_{h}\right\|_{2}^{2}+\tau \varepsilon_{2}\left\|\stackrel{*}{\delta^{2}} w_{h}\right\|_{2}^{2} .
$$

4.4 联合 (4.5) 与 (4.7) 式, 取 $\varepsilon_{2}=\frac{\sigma_{0}}{2 C}$ 和 $\varepsilon_{3}=\min \left(\frac{1}{8 C \Lambda}, \frac{1}{2 C}\right)$, 则当 $\tau \leqslant \min \left(\frac{\varepsilon_{2}}{8 C}, \frac{\varepsilon_{3}}{8}, 1\right)$ 时, 得

$$
\left\|\delta w_{h}\right\|_{2}^{2}+\tau \sigma_{0}\left\|\delta^{*}{ }^{2} w_{h}\right\|_{2}^{2} \leqslant 0 .
$$

所以, $w_{h} \equiv 0$, 即 $v_{h}^{n+1}=\bar{v}_{h}^{n+1}$. 因此, 得到如下关于差分格式 (2.4)-(2.6) 的唯一性定理:

定理 3 假设定理 1 的条件成立, 则存在仅依赖给定数据和 $\Lambda$ 的正常数 $\tau_{0}$, 当 $\tau \leqslant \tau_{0}$ 时, 差分格式 (2.4)-(2.6) 的解 $v_{\Delta}=v_{h}^{\tau}=\left\{v_{j}^{n} \mid j=0,1, \ldots, J ; n=0,1, \ldots, N\right\}$ 是唯一的.

\section{5 稳定性}

5.1 现在讨论差分格式 (2.4)-(2.6) 的无条件稳定性. 假定 $\tilde{v}_{\Delta}=\left\{\tilde{v}_{j}^{n} \mid j=0,1, \ldots, J ; n=\right.$ $0,1, \ldots, N\}$ 满足

$$
\begin{aligned}
& \frac{\tilde{v}_{j}^{n+1}-\tilde{v}_{j}^{n}}{\tau}=\tilde{A}_{j}^{n+1} \stackrel{*}{\delta}^{2} \tilde{v}_{j}^{n+1}+\tilde{f}_{j}^{n+1} \quad(j=1,2, \ldots, J-1 ; n=0,1, \ldots, N-1), \\
& \tilde{v}_{0}^{n+1}=\tilde{v}_{J}^{n+1}=0 \quad(n=0,1, \ldots, N-1), \\
& \tilde{v}_{j}^{0}=\tilde{\varphi}_{j} \quad(j=0,1, \ldots, J),
\end{aligned}
$$

其中

$$
\tilde{A}_{j}^{n+1}=\tilde{A}\left(x_{j}, t^{n+1}, \bar{\delta}^{0} v_{j}^{n+1}\right), \quad \tilde{f}_{j}^{n+1}=\tilde{f}\left(x_{j}, t^{n+1}, \hat{\delta}^{0} v_{j}^{n+1}, \bar{\delta}^{1} v_{j}^{n+1}\right) .
$$

设 $m \times m$ 系数矩阵 $\tilde{A}(x, t, u), m$ 维向量函数 $\tilde{f}(x, t, u, p)$ 和 $\tilde{\varphi}(x)$ 分别近似于 $A(x, t, u)$ 、 $f(x, t, u, p)$ 和 $\varphi(x)$, 它们也满足条件 (I)-(III), 则差

$$
w_{\Delta}=v_{\Delta}-\tilde{v}_{\Delta}=\left\{w_{j}^{n}=v_{j}^{n}-\tilde{v}_{j}^{n} \mid j=0,1, \ldots, J ; n=0,1, \ldots, N\right\}
$$

满足

$$
\frac{w_{j}^{n+1}-w_{j}^{n}}{\tau}=A_{j}^{n+1} \stackrel{*}{\delta}^{2} w_{j}^{n+1}+\left(A_{j}^{n+1}-\tilde{A}_{j}^{n+1}\right) \stackrel{*}{\delta}^{2} \tilde{v}_{j}^{n+1}+\left(f_{j}^{n+1}-\tilde{f}_{j}^{n+1}\right)
$$




$$
\begin{aligned}
& (j=1,2, \ldots, J-1 ; n=0,1, \ldots, N-1), \\
& w_{0}^{n+1}=w_{J}^{n+1}=0 \quad(n=0,1, \ldots, N-1), \\
& w_{j}^{0}=\varphi_{j}-\tilde{\varphi}_{j} \quad(j=0,1, \ldots, J) .
\end{aligned}
$$

方程 (5.4) 可改写成

$$
\frac{w_{j}^{n+1}-w_{j}^{n}}{\tau}=A_{j}^{n+1} \stackrel{*}{\delta}^{2} w_{j}^{n+1}+S_{j}^{n+1}+R_{j}^{n+1}
$$

其中

$$
\begin{aligned}
& S_{j}^{n+1}=B(v, \tilde{v})_{j}^{n+1} \bar{\delta}^{0} w_{j}^{n+1}+C(v, \tilde{v})_{j}^{n+1} \hat{\delta}^{0} w_{j}^{n+1}+D(v, \tilde{v})_{j}^{n+1} \bar{\delta}^{1} w_{j}^{n+1}, \\
& R_{j}^{n+1}=A[\tilde{v}]_{j}^{n+1} \stackrel{\delta}{*}^{2} \tilde{v}_{j}^{n+1}+F[\tilde{v}]_{j}^{n+1}, \\
& B(v, \tilde{v})_{j}^{n+1}=\left(\tilde{A}_{u}\right)_{j}^{n+1} \stackrel{\delta}{ }^{2} \tilde{v}_{j}^{n+1}, C(v, \tilde{v})_{j}^{n+1}=\left(\tilde{f}_{u}\right)_{j}^{n+1}, \quad D(v, \tilde{v})_{j}^{n+1}=\left(\tilde{f}_{p}\right)_{j}^{n+1},
\end{aligned}
$$

其中对 $\bar{\delta}^{0} w_{j}^{n+1} \neq 0$, 定义

$$
\left(\tilde{A}_{u}\right)_{j}^{n+1}=\frac{A\left(x_{j}, t^{n+1}, \bar{\delta}^{0} v_{j}^{n+1}\right)-A\left(x_{j}, t^{n+1}, \bar{\delta}^{0} \tilde{v}_{j}^{n+1}\right)}{\bar{\delta}^{0} w_{j}^{n+1}}
$$

对 $\bar{\delta}^{0} w_{j}^{n+1}=0$, 定义 $\left(\tilde{A}_{u}\right)_{j}^{n+1}=0$; 且对 $\hat{\delta}^{0} w_{j}^{n+1} \neq 0$,

$$
\left(\tilde{f}_{u}\right)_{j}^{n+1}=\frac{f\left(x_{j}, t^{n+1}, \hat{\delta}^{0} v_{j}^{n+1}, \bar{\delta}^{1} \tilde{v}_{j}^{n+1}\right)-f\left(x_{j}, t^{n+1}, \hat{\delta}^{0} \tilde{v}_{j}^{n+1}, \bar{\delta}^{1} \tilde{v}_{j}^{n+1}\right)}{\hat{\delta}^{0} w_{j}^{n+1}},
$$

对 $\hat{\delta}^{0} w_{j}^{n+1}=0$, 定义 $\left(\tilde{f}_{u}\right)_{j}^{n+1}=0$; 对 $\left(\tilde{f}_{p}\right)_{j}^{n+1}$ 有类似的表达式; 并且

$$
\begin{aligned}
& A[\tilde{v}]_{j}^{n+1}=A\left(x_{j}, t^{n+1}, \bar{\delta}^{0} \tilde{v}_{j}^{n+1}\right)-\tilde{A}\left(x_{j}, t^{n+1}, \bar{\delta}^{0} \tilde{v}_{j}^{n+1}\right), \\
& F[\tilde{v}]_{j}^{n+1}=f\left(x_{j}, t^{n+1}, \hat{\delta}^{0} \tilde{v}_{j}^{n+1}, \bar{\delta}^{1} \tilde{v}_{j}^{n+1}\right)-\tilde{f}\left(x_{j}, t^{n+1}, \hat{\delta}^{0} \tilde{v}_{j}^{n+1}, \bar{\delta}^{1} \tilde{v}_{j}^{n+1}\right) .
\end{aligned}
$$

5.2 用向量 $\delta^{*} w_{j}^{n+1} h \tau$ 与 $(5.7)$ 式作内积, 并对 $j=1,2, \ldots, J-1$ 求和, 与第 3 节论证类似, 可得

$$
\begin{aligned}
& \left\|\delta w_{h}^{n+1}\right\|_{2}^{2}-\left\|\delta w_{h}^{n}\right\|_{2}^{2}+2 \tau \sum_{j=1}^{J-1}\left({ }^{*}{ }^{2} w_{j}^{n+1}, A_{j}^{n+1} \stackrel{\delta}{ }^{2} w_{j}^{n+1}\right) h \\
& \quad+\tau r \sum_{j=1}^{J-1}\left(2-\lambda_{j-1}-\mu_{j+1}\right)\left(\left|\Delta_{\tau} v_{j}^{n+1}\right|^{2}-\left|\Delta_{\tau} v_{j}^{n}\right|^{2}\right) h \\
& \leqslant 2 \tau\left|\sum_{j=1}^{J-1}\left(\stackrel{*}{\delta}^{2} w_{j}^{n+1}, S_{j}^{n+1}+R_{j}^{n+1}\right) h\right| .
\end{aligned}
$$

由此, 可得

$$
\begin{aligned}
\left\|\delta w_{h}^{n+1}\right\|_{2}^{2}- & \left\|\delta w_{h}^{n}\right\|_{2}^{2}+\tau r \sum_{j=1}^{J-1}\left(2-\lambda_{j-1}-\mu_{j+1}\right)\left(\left|\Delta_{\tau} w_{j}^{n+1}\right|^{2}-\left|\Delta_{\tau} w_{j}^{n}\right|^{2}\right) h \\
& +\sigma_{0} \tau\left\|\delta^{*} w_{h}^{n+1}\right\|_{2}^{2} \leqslant \frac{4 \tau}{\sigma_{0}} \sum_{j=1}^{J-1}\left(\left|S_{j}^{n+1}\right|^{2}+\left|R_{j}^{n+1}\right|^{2}\right) h .
\end{aligned}
$$

由条件 $(\mathrm{I})-(\mathrm{II})$ 、定义 $(2.7)$ 和引理 2.3 , 有

$$
\sum_{j=1}^{J-1}\left|B_{j}^{n+1} \bar{\delta}^{0} w_{j}^{n+1}\right|^{2} h \leqslant C\left(\left\|w_{h}^{n-1}\right\|_{\infty}^{2}+\left\|w_{h}^{n}\right\|_{\infty}^{2}+\left\|w_{h}^{n+1}\right\|_{\infty}^{2}\right)\left\|^{*}{ }^{2} \tilde{v}_{h}^{n+1}\right\|_{2}^{2},
$$




$$
\begin{gathered}
\sum_{j=1}^{J-1}\left|C_{j}^{n+1} \hat{\delta}^{0} w_{j}^{n+1}\right|^{2} h \leqslant C\left(\left\|w_{h}^{n-1}\right\|_{2}^{2}+\left\|w_{h}^{n}\right\|_{2}^{2}+\left\|w_{h}^{n+1}\right\|_{2}^{2}\right), \\
\sum_{j=1}^{J-1}\left|D_{j}^{n+1} \bar{\delta}^{1} w_{j}^{n+1}\right|^{2} h \leqslant C\left(\left\|\delta w_{h}^{n-1}\right\|_{2}^{2}+\left\|\delta w_{h}^{n}\right\|_{2}^{2}+\left\|\delta w_{h}^{n+1}\right\|_{2}^{2}\right. \\
\left.+\Lambda \tau\left\|\Delta_{\tau} w_{h}^{n+1}\right\|_{2}^{2}+\Lambda \tau\left\|\Delta_{\tau} w_{h}^{n}\right\|_{2}^{2}\right), \\
\sum_{j=1}^{J-1}\left|R_{j}^{n+1}\right|^{2} h \leqslant C\left(\left\|A[\tilde{v}]_{h}^{n+1}\right\|_{\infty}\left\|\stackrel{*}{\delta}^{2} \tilde{v}_{h}^{n+1}\right\|_{2}^{2}+\left\|F[\tilde{v}]_{h}^{n+1}\right\|_{2}^{2}\right),
\end{gathered}
$$

其中 $C$ 为不依赖步长 $h$ 和 $\tau$ 的常数.

将上面的不等式代入 (5.8) 式, 得

$$
\begin{gathered}
\left\|\delta w_{h}^{n+1}\right\|_{2}^{2}-\left\|\delta w_{h}^{n}\right\|_{2}^{2}+\tau r \sum_{j=1}^{J-1}\left(2-\lambda_{j-1}-\mu_{j+1}\right)\left(\left|\Delta_{\tau} w_{j}^{n+1}\right|^{2}-\left|\Delta_{\tau} w_{j}^{n}\right|^{2}\right) h+\sigma_{0} \tau\left\|^{*}{ }^{2} w_{h}^{n+1}\right\|_{2}^{2} \\
\leqslant C \tau\left\{\left\|\delta w_{h}^{n-1}\right\|_{2}^{2}+\left\|\delta w_{h}^{n}\right\|_{2}^{2}+\left\|\delta w_{h}^{n+1}\right\|_{2}^{2}+\left(\left\|w_{h}^{n-1}\right\|_{\infty}^{2}+\left\|w_{h}^{n}\right\|_{\infty}^{2}+\left\|w_{h}^{n+1}\right\|_{\infty}^{2}\right)\left\|\delta^{2} \tilde{v}_{h}^{n+1}\right\|_{2}^{2}\right. \\
\left.+\Lambda \tau\left\|\Delta_{\tau} w_{h}^{n+1}\right\|_{2}^{2}+\Lambda \tau\left\|\Delta_{\tau} w_{h}^{n}\right\|_{2}^{2}+\left\|A[\tilde{v}]_{h}^{n+1}\right\|_{\infty}^{2}\left\|\delta^{*}{ }^{2} \tilde{v}_{h}^{n+1}\right\|_{2}^{2}+\left\|F[\tilde{v}]_{h}^{n+1}\right\|_{2}^{2}\right\} .
\end{gathered}
$$

5.3 当 $n \geqslant 1$, 由 (5.7) 式得

$$
\begin{aligned}
\tau\left\|\Delta_{\tau} w_{h}^{n+1}\right\|_{2}^{2} \leqslant & C \tau\left\{\left\|\stackrel{*}{\delta}^{2} w_{h}^{n+1}\right\|_{2}^{2}+\left(\left\|w_{h}^{n-1}\right\|_{\infty}^{2}+\left\|w_{h}^{n}\right\|_{\infty}^{2}+\left\|w_{h}^{n+1}\right\|_{\infty}^{2}\right)\left\|\stackrel{*}{\delta}^{2} \tilde{v}_{h}^{n+1}\right\|_{2}^{2}\right. \\
& +\left\|\delta w_{h}^{n-1}\right\|_{2}^{2}+\left\|\delta w_{h}^{n}\right\|_{2}^{2}+\left\|\delta w_{h}^{n+1}\right\|_{2}^{2}+\Lambda \tau\left\|\Delta_{\tau} w_{h}^{n}\right\|_{2}^{2}+\Lambda \tau\left\|\Delta_{\tau} w_{h}^{n+1}\right\|_{2}^{2} \\
& \left.+\left\|A[\tilde{v}]_{h}^{n+1}\right\|_{\infty}^{2}\left\|\stackrel{*}{\delta}^{2} \tilde{v}_{h}^{n+1}\right\|_{2}^{2}+\left\|F[\tilde{v}]_{h}^{n+1}\right\|_{2}^{2}\right\} .
\end{aligned}
$$

取 $\tau>0$, 使得 $C \Lambda \tau \leqslant \frac{1}{2}$, 则

$$
\sum_{k=0}^{n}\left\|\Delta_{\tau} w_{h}^{k+1}\right\|_{2}^{2} \tau \leqslant C\left(\sum_{k=0}^{n}\left\|\delta^{*} w_{h}^{n+1}\right\|_{2}^{2} \tau+\max _{0 \leqslant k \leqslant n+1}\left\|w_{h}^{k}\right\|_{\infty}^{2}+\sum_{k=0}^{n+1}\left\|\delta w_{h}^{k}\right\|_{2}^{2} \tau\right) .
$$

5.4 由 (5.9) 式可得

$$
\begin{gathered}
\left\|\delta w_{h}^{n+1}\right\|_{2}^{2}+\sigma_{0} \sum_{k=0}^{n}\left\|\delta^{*}{ }^{2} w_{h}^{k+1}\right\|_{2}^{2} \tau+\tau r \sum_{j=1}^{J-1}\left(2-\lambda_{j-1}-\mu_{j+1}\right)\left|\Delta_{\tau} w_{j}^{n+1}\right|^{2} h \\
\leqslant C\left(\sum_{k=0}^{n}\left\|\delta w_{h}^{k+1}\right\|_{2}^{2} \tau+\max _{0 \leqslant k \leqslant n+1}\left\|w_{h}^{k}\right\|_{\infty}^{2}+I_{0}\right)+C \tau \sum_{k=0}^{n}\left\|\Delta_{\tau} w_{h}^{k+1}\right\|_{2}^{2} \tau .
\end{gathered}
$$

其中

$$
I_{0} \equiv\left\|\delta w_{h}^{0}\right\|_{2}^{2}+\max _{0 \leqslant n \leqslant N-1}\left\|A[\tilde{v}]_{h}^{n+1}\right\|_{\infty}^{2}+\sum_{n=0}^{N-1}\left\|F[\tilde{v}]_{h}^{n+1}\right\|_{2}^{2} \tau .
$$

利用 (5.10) 式, 并取 $C \varepsilon<\frac{\sigma_{0}}{4}$, 得

$$
\begin{gathered}
\left\|\delta w_{h}^{n+1}\right\|_{2}^{2}+\frac{3 \sigma_{0}}{4} \sum_{k=0}^{n}\left\|\stackrel{\delta}{\delta}^{2} w_{h}^{k+1}\right\|_{2}^{2} \tau+\tau r \sum_{j=1}^{J-1}\left(2-\lambda_{j-1}-\mu_{j+1}\right)\left|\Delta_{\tau} w_{j}^{n+1}\right|^{2} h+\varepsilon \sum_{k=0}^{n}\left\|\Delta_{\tau} w_{h}^{k+1}\right\|_{2}^{2} \tau \\
\leqslant C\left(\sum_{k=0}^{n}\left\|\delta w_{h}^{k+1}\right\|_{2}^{2} \tau+\max _{0 \leqslant k \leqslant n+1}\left\|w_{h}^{k}\right\|_{\infty}^{2}+I_{0}\right)+C \tau \sum_{k=0}^{n}\left\|\Delta_{\tau} w_{h}^{k+1}\right\|_{2}^{2} \tau
\end{gathered}
$$


将 (5.10) 式代入 (5.11) 式的右端, 并取 $C \tau<\frac{\sigma_{0}}{4}$, 得

$$
\begin{aligned}
\left\|\delta w_{h}^{n+1}\right\|_{2}^{2}+ & \frac{\sigma_{0}}{2} \sum_{k=0}^{n}\left\|\delta^{*} w_{h}^{k+1}\right\|_{2}^{2} \tau+\tau r \sum_{j=1}^{J-1}\left(2-\lambda_{j-1}-\mu_{j+1}\right)\left|\Delta_{\tau} w_{j}^{n+1}\right|^{2} h+\varepsilon \sum_{k=0}^{n}\left\|\Delta w_{h}^{k+1}\right\|_{2}^{2} \tau \\
& \leqslant C\left(\sum_{k=0}^{n}\left\|\delta w_{h}^{k+1}\right\|_{2}^{2} \tau+\max _{0 \leqslant k \leqslant n+1}\left\|w_{h}^{k}\right\|_{\infty}^{2}+I_{0}\right) .
\end{aligned}
$$

5.5 由上式及引理 2.2 , 对 $0 \leqslant n \leqslant N-1$, 有

$$
\begin{aligned}
\left\|\delta w_{h}^{n+1}\right\|_{2}^{2}+\frac{\sigma_{0}}{2} \sum_{k=0}^{n}\left\|{ }^{*}{ }^{2} w_{h}^{k+1}\right\|_{2}^{2} \tau & +\tau r \sum_{j=1}^{J-1}\left(2-\lambda_{j-1}-\mu_{j+1}\right)\left|\Delta_{\tau} w_{j}^{n+1}\right|^{2} h+\varepsilon \sum_{k=0}^{n}\left\|\Delta_{\tau} w_{h}^{k+1}\right\|_{2}^{2} \tau \\
& \leqslant C\left(\max _{0 \leqslant k \leqslant n+1}\left\|w_{h}^{k}\right\|_{\infty}^{2}+I_{0}\right)
\end{aligned}
$$

由引理 2.3 , 对任意 $\varepsilon_{4}>0$, 有

$$
\begin{gathered}
\left\|\delta w_{h}^{n+1}\right\|_{2}^{2}+\frac{\sigma_{0}}{2} \sum_{k=0}^{n}\left\|\delta^{*}{ }^{2} w_{h}^{k+1}\right\|_{2}^{2} \tau+\varepsilon \sum_{k=0}^{n}\left\|\Delta_{\tau} w_{h}^{k+1}\right\|_{2}^{2} \tau \\
\leqslant C\left(\varepsilon_{4} \max _{0 \leqslant k \leqslant n+1}\left\|\delta w_{h}^{k}\right\|_{2}^{2}+\frac{1}{\varepsilon_{4}} \max _{0 \leqslant k \leqslant n+1}\left\|w_{h}^{k}\right\|_{2}^{2}+I_{0}\right) .
\end{gathered}
$$

\section{6 如下引理显然成立:}

引理 5.1 如果离散函数 $D(k)$ 和 $G(k)(0 \leqslant k \leqslant N)$ 满足 $G(k) \leqslant G(k+1)(0 \leqslant k \leqslant$ $N-1)$, 且

$$
D(n) \leqslant \frac{1}{2} \max _{0 \leqslant k \leqslant n} D(k)+G(n), \quad n=0,1, \ldots, N,
$$

则 $D(n) \leqslant 2 G(n), n=0,1, \ldots, N$.

引理 5.2 对任意 $\varepsilon_{5}>0, n=0,1, \ldots, N-1$,

$$
\max _{0 \leqslant k \leqslant n}\left\|w_{h}^{k+1}\right\|_{2}^{2} \leqslant \varepsilon_{5} \sum_{k=0}^{n}\left\|\Delta_{\tau} w_{h}^{k+1}\right\|_{2}^{2} \tau+\frac{1}{\varepsilon_{5}} \sum_{k=0}^{n+1}\left\|w_{h}^{k}\right\|_{2}^{2} \tau+\left\|w_{h}^{0}\right\|_{2}^{2} .
$$

5.7 在 (5.13) 式中令 $C \varepsilon_{4} \leqslant \frac{1}{2}$, 并利用引理 5.1 , 得

$$
\left\|\delta w_{h}^{n+1}\right\|_{2}^{2}+\frac{\sigma_{0}}{2} \sum_{k=0}^{n}\left\|\delta^{*}{ }^{2} w_{h}^{k+1}\right\|_{2}^{2} \tau+\varepsilon \sum_{k=0}^{n}\left\|\Delta_{\tau} w_{h}^{k+1}\right\|_{2}^{2} \tau \leqslant C\left(\max _{0 \leqslant k \leqslant n+1}\left\|w_{h}^{k}\right\|_{2}^{2}+I_{0}\right) .
$$

利用引理 5.2 , 并取 $C \varepsilon_{5} \leqslant \frac{\varepsilon}{2}$, 对 $0 \leqslant n \leqslant N-1$, 有

$$
\left\|\delta w_{h}^{n+1}\right\|_{2}^{2}+\frac{\sigma_{0}}{2} \sum_{k=0}^{n}\left\|\delta^{*}{ }^{2} w_{h}^{k+1}\right\|_{2}^{2} \tau+\frac{\varepsilon}{2} \sum_{k=0}^{n}\left\|\Delta_{\tau} w_{h}^{k+1}\right\|_{2}^{2} \tau \leqslant C\left(\sum_{k=0}^{n+1}\left\|w_{h}^{k}\right\|_{2}^{2} \tau+I_{0}\right) .
$$

于是, 由引理 2.2 (ii), 对 $n=0,1, \ldots, N-1$, 得

$$
\left\|\delta w_{h}^{n+1}\right\|_{2}^{2}+\sum_{k=0}^{n}\left\|\delta^{*}{ }^{2} w_{h}^{k+1}\right\|_{2}^{2} \tau+\sum_{k=0}^{n}\left\|\Delta_{\tau} w_{h}^{k+1}\right\|_{2}^{2} \tau \leqslant C I_{0} .
$$

5.8 这表明并行格式 (2.4)- (2.6) 的解 $v_{\Delta}$ 在离散泛函空间 $W_{2}^{(2,1)}\left(Q_{\Delta}\right)$ 连续依赖于离散初值 函数 $\varphi(x)$ 在离散 $H^{1}$ 空间的范数、系数矩阵 $A(x, t, u)$ 在离散 $L^{\infty}$ 空间的范数以及非线性函 数 $f(x, t, u, p)$ 在离散 $L^{2}$ 空间的范数. 于是, 证明了如下稳定性定理: 
定理 4 在定理 1 的条件下, 对 $w_{\Delta}=v_{\Delta}-\tilde{v}_{\Delta}=\left\{w_{j}^{n}=v_{j}^{n}-\tilde{v}_{j}^{n} \mid j=0,1, \ldots, J ; n=\right.$ $0,1, \ldots, N\}$ 成立如下不等式:

$$
\begin{aligned}
& \left\|v_{\Delta}-\tilde{v}_{\Delta}\right\|_{W_{2}^{(2,1)}\left(Q_{\Delta}\right)}^{2} \\
& \leqslant K\left\{\left\|\varphi_{h}-\tilde{\varphi}_{h}\right\|_{H_{h}^{1}}^{2}+\max _{|u| \leqslant C} \max _{1 \leqslant j \leqslant J-1,0 \leqslant n \leqslant N-1}\left|A\left(x_{j}, t^{n+1}, u\right)-\tilde{A}\left(x_{j}, t^{n+1}, u\right)\right|^{2}\right. \\
& \left.\quad+\max _{|u| \leqslant C,|p|<\infty} \sum_{j=1}^{J-1} \sum_{n=0}^{N-1}\left|f\left(x_{j}, t^{n+1}, u, p\right)-\tilde{f}\left(x_{j}, t^{n+1}, u, p\right)\right|^{2} h \tau\right\},
\end{aligned}
$$

其中 $K$ 为不依赖步长 $h$ 和 $\tau$ 的常数, 常数 $C$ 由 (3.9) 式确定, 且

$$
\begin{aligned}
& \left\|\varphi_{h}\right\|_{H_{h}^{1}}^{2}=\left\|\varphi_{h}\right\|_{2}^{2}+\left\|\delta \varphi_{h}\right\|_{2}^{2}, \\
& \left\|w_{\Delta}\right\|_{W_{2}^{(2,1)}\left(Q_{\Delta}\right)}^{2} \equiv \max _{n=0,1, \ldots, N}\left\|w_{h}^{n}\right\|_{H_{h}^{1}}^{2}+\sum_{n=0}^{N-1}\left\|\stackrel{*}{\delta^{2}} w_{h}^{n+1}\right\|_{2}^{2} \tau+\sum_{n=0}^{N-1}\left\|\Delta_{\tau} w_{h}^{n+1}\right\|_{2}^{2} \tau .
\end{aligned}
$$

\section{6 收敛性}

6.1 本节利用已得到的估计式将证明差分格式 (2.4)-(2.6) 的解 $v_{\Delta}=v_{h}^{\tau}=\left\{v_{j}^{n} \mid j=0,1, \ldots, J\right.$; $n=0,1, \ldots, N\}$ 的收玫性.

引理 6.1 对差分格式 (2.4)-(2.6) 的离散解, 有

$$
\begin{aligned}
& \max _{n=0,1, \ldots, N}\left|v_{j}^{n}-v_{j^{\prime}}^{n}\right| \leqslant C\left|x_{j}-x_{j^{\prime}}\right|^{\frac{1}{2}}, \quad 0 \leqslant j, j^{\prime} \leqslant J ; \\
& \max _{j=0,1, \ldots, J}\left|v_{j}^{n}-v_{j}^{n^{\prime}}\right| \leqslant C\left|t^{n}-t^{n^{\prime}}\right|^{\frac{1}{4}}, \quad 0 \leqslant n, n^{\prime} \leqslant N ; \\
& \left(\sum_{n=0}^{N-1} \sum_{j=0}^{J-m-1}\left|\delta v_{j+m}^{n}-\delta v_{j}^{n}\right|^{2} h \tau\right)^{\frac{1}{2}} \leqslant C m h, \\
& \left(\sum_{n=0}^{N-s} \sum_{j=0}^{J-1}\left|\delta v_{j}^{n+s}-\delta v_{j}^{n}\right|^{2} h \tau\right)^{\frac{1}{2}} \leqslant C(s \tau)^{\frac{1}{4}},
\end{aligned}
$$

其中 $m$ 和 $s$ 是满足 $0 \leqslant m \leqslant J-1,0 \leqslant s \leqslant N$ 的整数; 且常数 $C$ 不依赖 $h, \tau, m, s$ 和 $v_{h}^{\tau}$.

证明 易见

$$
\left|v_{j}^{n}-v_{j^{\prime}}^{n}\right| \leqslant\left\|\delta v_{h}^{n}\right\|_{2}\left|x_{j}-x_{j^{\prime}}\right|^{\frac{1}{2}},
$$

由 (3.7) 式即得估计 (6.1) . 由引理 2.3 和 (3.7) 及 (3.8) 式, 有

$$
\left\|v_{h}^{n}-v_{h}^{n^{\prime}}\right\|_{\infty} \leqslant\left\|\delta\left(v_{h}^{n}-v_{h}^{n^{\prime}}\right)\right\|_{2}^{\frac{1}{2}}\left\|v_{h}^{n}-v_{h}^{n^{\prime}}\right\|_{2}^{\frac{1}{2}} \leqslant C\left\|v_{h}^{n}-v_{h}^{n^{\prime}}\right\|_{2}^{\frac{1}{2}},
$$

并且

$$
\left\|v_{h}^{n}-v_{h}^{n^{\prime}}\right\|_{2} \leqslant\left(\sum_{k=0}^{N-1}\left\|\Delta_{\tau} v_{h}^{k+1}\right\|_{2}^{2} \tau\right)^{\frac{1}{2}}\left|t^{n}-t^{n^{\prime}}\right|^{\frac{1}{2}} \leqslant C\left|t^{n}-t^{n^{\prime}}\right|^{\frac{1}{2}},
$$

由此, 可得 (6.2) 式. 类似地, 可由 (3.9) 式推得 (6.3) 式. 注意

$$
\left\|\delta v_{h}^{n+s}-\delta v_{h}^{n}\right\|_{2}^{2} \leqslant C\left\|\delta^{2} v_{h}^{n+s}-\delta^{2} v_{h}^{n}\right\|_{2}\left\|v_{h}^{n+s}-v_{h}^{n}\right\|_{2} \leqslant C(s \tau)^{\frac{1}{2}},
$$


可见 (6.4) 式成立. 引理证毕.

6.2 对 $(x, t) \in Q_{j}^{n}(j=0,1, \ldots, J-1 ; n=0,1, \ldots, N-1)$ 定义分片常数函数

$$
v_{h}^{\tau}(x, t)=v_{j}^{n+1}, \quad v_{x h}^{\tau}(x, t)=\delta v_{j}^{n+1}=\frac{1}{2}\left(\delta v_{j+\frac{1}{2}}^{n+1}+v_{j-\frac{1}{2}}^{n+1}\right), \quad v_{t h}^{\tau}(x, t)=\Delta_{\tau} v_{j}^{n+1} .
$$

并定义

$$
\begin{array}{ll}
v_{x x h}^{\tau}(x, t)=\delta^{*}{ }^{2} v_{j}^{n+1}, & (x, t) \in Q_{j}^{n} \quad(j=1,2, \ldots, J-1 ; n=0,1, \ldots, N-1), \\
v_{x x h}^{\tau}(x, t)=\delta^{*} v_{1}^{n+1}, \quad(x, t) \in Q_{0}^{n} \quad(n=0,1, \ldots, N-1) .
\end{array}
$$

记 $J_{0}$ 为满足 $\lambda_{j} \neq 1$ 或 $\mu_{j} \neq 1$ 的下标 $j$ 的个数, 即不是纯隐式的网格点的个数. 引入如 下条件 $(\mathrm{V})$, 它表明不为纯隐式格式的空间点的个数不超过一个固定常数, 即区域分解后所得 到的 (纯隐式) 子区域的个数对所有 $h>0$ 和 $\tau>0$ 是一致有界的.

(V) 对所有 $h>0$ 和 $\tau>0, J_{0}$ 不超过任一固定常数.

引理 6.2 假设条件 $(\mathrm{V})$ 和定理 1 的条件成立, 则当 $h \rightarrow 0, \tau \rightarrow 0$ 时 (对某个子列), 存 在函数 $u(x, t) \in W_{2}^{(2,1)}\left(Q_{T}\right)$, 使得

(i) $v_{h}^{\tau}(x, t) \rightarrow u(x, t)$ 在 $Q_{T}$ 中一致收敛;

(ii) $v_{x h}^{\tau}(x, t) \rightarrow u_{x}(x, t)$ 在 $L^{2}\left(Q_{T}\right)$ 中强收玫, 在 $Q_{T}$ 中几乎处处收玫;

(iii) $v_{x x h}^{\tau}(x, t) \rightarrow u_{x x}(x, t)$ 在 $L^{2}\left(Q_{T}\right)$ 中弱收玫;

(iv) $v_{t h}^{\tau}(x, t) \rightarrow u_{t}(x, t)$ 在 $L^{2}\left(Q_{T}\right)$ 中弱收玫.

证明 利用引理 6.1 和文献 [11] 中离散紧性方法, 由 $v_{h}^{\tau}(x, t)$ 构造的双线性插值函数, 可 以证明 (i) 和 (ii).

由估计 (3.8) 以及 $L^{2}$ 中准紧集判别准则, 得函数 $u^{\prime \prime}(x, t) \in L^{2}\left(Q_{T}\right)$, 使得 $v_{x x h}^{\tau}(x, t) \rightarrow$ $u^{\prime \prime}(x, t)$ 在 $L^{2}\left(Q_{T}\right)$ 中弱收玫. 下面证明 $u^{\prime \prime}(x, t)=u_{x x}(x, t)$ 在 $Q_{T}$ 中成立. 令 $\Phi(x, t) \in$ $C_{0}^{\infty}\left(Q_{T}\right)$. 记 $\Phi_{j}^{n}=\Phi\left(x_{j}, t^{n}\right)$. 定义分片常数函数 $\Phi_{h}^{\tau}(x, t)=\Phi_{j}^{n+1}, \Phi_{x x h}^{\tau}(x, t)=\delta^{2} \Phi_{j}^{n+1}$, $(x, t) \in Q_{j}^{n}$. 当 $h$ 和 $\tau$ 足够小时,

$$
\begin{aligned}
\int & \int_{Q_{T}} v_{x x h}^{\tau}(x, t) \Phi_{h}^{\tau}(x, t) d x d t \\
= & \sum_{n=0}^{N-1} \sum_{j=1}^{J-1} \delta^{2} v_{j}^{n+1} \Phi_{j}^{n+1} h \tau=\sum_{n=0}^{N-1} \sum_{j=1}^{J-1} \delta^{2} v_{j}^{n+1} \Phi_{j}^{n+1} h \tau \\
& -r \sum_{n=0}^{N-1} \sum_{j=1}^{J-1} \frac{\left(1-\lambda_{j}\right)\left(\Delta_{\tau} v_{j+1}^{n+1}-\Delta_{\tau} v_{j+1}^{n}\right)+\left(1-\mu_{j}\right)\left(\Delta_{\tau} v_{j-1}^{n+1}-\Delta_{\tau} v_{j-1}^{n}\right)}{h} \Phi_{j}^{n+1} h \tau \\
\equiv & \mathrm{I}+\mathrm{II} .
\end{aligned}
$$

易见

$$
\begin{aligned}
& \mathrm{I} \rightarrow \iint_{Q_{T}} u(x, t) \Phi_{x x}(x, t) d x d t, \quad h \rightarrow 0, \tau \rightarrow 0 ; \\
& |\mathrm{II}| \leqslant 4 \Lambda \max _{0 \leqslant n \leqslant N-1}\left\|\Phi_{h}^{n+1}\right\|_{\infty}\left(\sum_{n=0}^{N-1}\left\|\Delta_{\tau} v_{h}^{n+1}\right\|_{2}^{2} \tau\right)^{\frac{1}{2}}\left(T J_{0} h\right)^{\frac{1}{2}},
\end{aligned}
$$

所以, 当 $h \rightarrow 0, \tau \rightarrow 0$ 时, II $\rightarrow 0$;

于是 (iii) 成立. 类似地 (iv) 成立. 引理证毕. 
6.3 对 $(x, t) \in Q_{j}^{n}(j=0,1, \ldots, J-1 ; n=0,1, \ldots, N-1)$, 定义分片常数函数

$$
\begin{aligned}
& \bar{v}_{h}^{\tau}(x, t)=\bar{\delta}^{0} v_{j}^{n+1}, \quad \hat{v}_{h}^{\tau}(x, t)=\hat{\delta}^{0} v_{j}^{n+1}, \quad \bar{v}_{x h}^{\tau}(x, t)=\bar{\delta}^{1} v_{j}^{n+1}, \\
& A_{h}^{\tau}(x, t)=A_{j}^{n+1}, \quad f_{h}^{\tau}(x, t)=f_{j}^{n+1} .
\end{aligned}
$$

引理 6.3 假设定理 1 的条件成立. 当 $h \rightarrow 0, \tau \rightarrow 0$ 时 (对某个子列), 有

(i) $\bar{v}_{h}^{\tau}(x, t) \rightarrow u(x, t), \hat{v}_{h}^{\tau}(x, t) \rightarrow u(x, t)$ 在 $L^{2}\left(Q_{T}\right)$ 中强收玫, 在 $Q_{T}$ 中几乎处处收玫;

(ii) $\bar{v}_{x h}^{\tau}(x, t) \rightarrow u_{x}(x, t)$ 在 $L^{2}\left(Q_{T}\right)$ 中强收玫, 在 $Q_{T}$ 中几乎处处收敛;

(iii) $A_{h}^{\tau}(x, t) \rightarrow A(x, t, u(x, t)), Q_{T} f_{h}^{\tau}(x, t) \rightarrow f\left(x, t, u(x, t), u_{x}(x, t)\right)$ 在 $L^{2}\left(Q_{T}\right)$ 中强收 敛, 在 $Q_{T}$ 中几乎处处收玫.

证明 由定义 (2.7) 和估计 (3.7) 及 (3.8), 可得

$$
\begin{aligned}
& \left\|\bar{v}_{h}^{\tau}(x, t)-v_{h}^{\tau}(x, t)\right\|_{L^{2}\left(Q_{T}\right)}^{2} \leqslant C h^{2} \sum_{n=0}^{N}\left\|\delta v_{h}^{n}\right\|_{2}^{2} \tau+C \tau^{2} \sum_{n=0}^{N-1}\left\|\Delta_{\tau} v_{h}^{n+1}\right\|_{2}^{2} \tau \leqslant C\left(h^{2}+\tau^{2}\right), \\
& \int_{0}^{T}\left\|\bar{v}_{x h}^{\tau}(x, t)-v_{x h}^{\tau}(x, t)\right\|_{2}^{2} d t \\
& \quad \leqslant C\left(\frac{\tau}{h}\right)^{2} \sum_{j=1}^{J-1} \sum_{n=0}^{N-1}\left|\Delta_{\tau} v_{j}^{n+1}\right|^{2} h \tau+C h^{2} \sum_{j=1}^{J-1} \sum_{n=0}^{N-1}\left|\delta^{2} v_{j}^{n+1}\right|^{2} h \tau \leqslant C\left(\Lambda \tau+h^{2}\right) .
\end{aligned}
$$

利用引理 6.2 以及上面两个不等式可推出 (i) 和 (ii). 现在证明 (iii). 由条件(I), 可知 $A(x, t, u) \in$ $C\left(Q_{T} \times \mathbb{R}^{m}\right)$, 且由 $(3.7)$ 式得 $\max _{0 \leqslant n \leqslant N-1}\left\|\bar{\delta}^{0} v_{h}^{n+1}\right\|_{\infty} \leqslant C$. 有

$$
\begin{aligned}
& \left\|A_{h}^{\tau}(x, t)-A(x, t, u(x, t))\right\|_{L^{2}\left(Q_{T}\right)}^{2} \\
& =\sum_{n=0}^{N-1} \sum_{j=0}^{J-1} \iint_{Q_{j}^{n}}\left|A\left(x_{j}, t^{n+1}, \bar{\delta}^{0} v_{j}^{n+1}\right)-A(x, t, u(x, t))\right|^{2} d x d t \\
& \leqslant 2 l T \max _{\left|x^{\prime}-x\right| \leqslant h,\left|t^{\prime}-t\right| \leqslant \tau,|v| \leqslant C}\left|A\left(x^{\prime}, t^{\prime}, v\right)-A(x, t, v)\right|^{2} \\
& \quad+2 C\left\|\bar{v}_{h}^{\tau}(x, t)-u(x, t)\right\|_{L^{2}\left(Q_{T}\right)}^{2},
\end{aligned}
$$

所以, $A_{h}^{\tau} \rightarrow A$ 在 $L^{2}\left(Q_{T}\right)$ 中强收玫. 类似地可得 $f_{h}^{\tau} \rightarrow f$ 在 $L^{2}\left(Q_{T}\right)$ 中强收玫. 引理证毕.

6.4 令 $\Phi(x, t) \in C^{\infty}\left(Q_{T}\right)$, 在 $x=0$ 和 $x=l$ 附近 $\Phi(x, t)=0$. 记 $\Phi_{j}^{n}=\Phi\left(x_{j}, t^{n}\right)$. 对 $(x, t) \in Q_{j}^{n}$, 定义分片常数函数 $\Phi_{h}^{\tau}(x, t)=\Phi_{j}^{n+1}$. 有

$$
\begin{aligned}
\int & \int_{Q_{T}}\left[v_{t h}^{\tau}(x, t)-A_{h}^{\tau}(x, t) v_{x x h}^{\tau}-f_{h}^{\tau}(x, t)\right] \Phi_{h}^{\tau}(x, t) d x d t \\
= & \sum_{n=0}^{N-1} \sum_{j=1}^{J-1}\left[\frac{v_{j}^{n+1}-v_{j}^{n}}{\tau}-A_{j}^{n+1} \delta^{*}{ }^{2} v_{j}^{n+1}-f_{j}^{n+1}\right] \Phi_{j}^{n+1} h \tau=0 .
\end{aligned}
$$

令 $h \rightarrow 0, \tau \rightarrow 0$ (对某个子列), 得

$$
\iint_{Q_{T}}\left[u_{t}(x, t)-A(x, t, u) u_{x x}(x, t)-f(x, t, u)\right] \Phi(x, t) d x d t=0 .
$$

由于子列 $v_{h}^{\tau}(x, t)$ 在 $Q_{T}$ 中一致收玫于 $u(x, t)$, 所以 $u(x, t)$ 满足齐次边界条件 $(2.2)$ 和初 值条件 (2.3). 这说明 $u(x, t) \in W_{2}^{(2,1)}\left(Q_{T}\right)$ 正是 $(2.1)-(2.3)$ 式的广义解, 不难验证 $(2.1)-(2.3)$ 式的广义解是唯一的 (见文献 [11]). 于是, 得到如下收玫性定理:

定理 5 假设定理 1 的条件成立. 那么, 当步长 $h$ 和 $\tau$ 趋于 $0,(2.4)-(2.6)$ 式的解 $v_{\Delta}=v_{h}^{\tau}=\left\{v_{j}^{n} \mid j=0,1, \ldots, J ; n=0,1, \ldots, N\right\}$ 收敛于 (2.1)-(2.3) 式的唯一广义解 $u(x, t) \in$ 
$W_{2}^{(2,1)}\left(Q_{T}\right)$.

\section{7 数值实验}

考虑问题

$$
\begin{aligned}
& u_{t}=u u_{x x}+f\left(x, t, u, u_{x}\right), \quad x \in[0,1], t \in[0, T], \\
& u(0, t)=u(1, t)=0, \quad t \in(0, T], \\
& u(x, 0)=\sin (\pi x), \quad x \in[0,1],
\end{aligned}
$$

其中 $f\left(x, t, u, u_{x}\right)=\pi^{2}\left(e^{-\pi^{2} t} \sin (\pi x)\right)^{2}+\pi e^{-\pi^{2} t} \cos (\pi x)-\pi^{2} u-u_{x}$. 该问题的精确解是 $u=$ $e^{-\pi^{2} t} \sin (\pi x)$, 在表 1 中我们给出了格式的精度实验结果.

其中 $\tau=0.0000001$, 计算 100000 个时间步长, 使用了 4 个处理机, $R_{1}$ 和 $R_{2}$ 是理论 值, $R$ 是计算值, 具体意义如下: 记 $E_{\infty}^{J-1}=\max _{\substack{0 \leqslant j \leqslant J-1 \\ 0 \leqslant \leqslant \leqslant N}}\left|v_{j}^{n}-u_{j}^{n}\right|, h_{J-1}=\frac{1}{J-1}$. 格式如 果是一阶精度的, 即存在与 $\tau, h_{J-1}$ 无关的常数 $C_{1}$, 使得 $E_{\infty}^{J-1} \approx C_{1}\left(\tau+h_{J-1}\right)$, 记 $R_{1}=$ $\frac{E_{\infty}^{10}}{E_{\infty}^{J-1}} \approx \frac{\tau+h_{10}}{\tau+h_{J-1}} \approx \frac{h_{10}}{h_{J-1}}=\frac{J-1}{10}$. 格式如果是二阶精度的, 即有 $E_{\infty}^{J-1} \approx C_{2}\left(\tau+h_{J-1}^{2}\right)$, 记

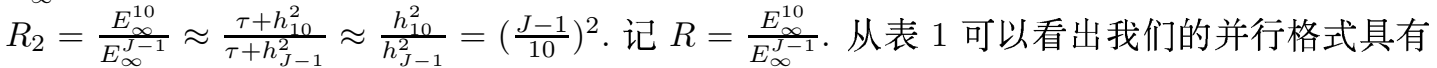
二阶精度.

表 1 格式的精度

\begin{tabular}{cccccc}
\hline $\mathrm{J}-1$ & 20 & 40 & 60 & 80 & 100 \\
\hline $\max _{j, n}\left|v_{j}^{n}-u_{j}^{n}\right|$ & $1.7468 \mathrm{E}-004$ & $4.3880 \mathrm{E}-005$ & $1.9516 \mathrm{E}-005$ & $1.0997 \mathrm{E}-005$ & $7.0508 \mathrm{E}-006$ \\
$\max _{j, n} \frac{\left|v_{j}^{n}-u_{j}^{n}\right|}{\left|u_{j}^{n}\right|}$ & $6.4136 \mathrm{E}-004$ & $2.8055 \mathrm{E}-004$ & $1.7093 \mathrm{E}-004$ & $1.1834 \mathrm{E}-004$ & $8.8271 \mathrm{E}-005$ \\
$R_{1}$ & - & 2 & 3 & 4 & 5 \\
$R_{2}$ & - & 4 & 9 & 16 & 25 \\
$\mathrm{R}$ & - & 3.98 & 8.95 & 15.88 & 24.77 \\
\hline \hline $\mathrm{J}-1$ & 120 & 140 & 160 & 180 & 200 \\
\hline $\max _{j, n}\left|v_{j}^{n}-u_{j}^{n}\right|$ & $4.9082 \mathrm{E}-006$ & $3.6155 \mathrm{E}-006$ & $2.7769 \mathrm{E}-006$ & $2.2018 \mathrm{E}-006$ & $1.7905 \mathrm{E}-006$ \\
$\max _{j, n} \frac{\left|v_{j}^{n}-u_{j}^{n}\right|}{\left|u_{j}^{n}\right|}$ & $6.9135 \mathrm{E}-005$ & $5.6055 \mathrm{E}-005$ & $4.6640 \mathrm{E}-005$ & $3.9593 \mathrm{E}-005$ & $3.4154 \mathrm{E}-005$ \\
$R_{1}$ & 6 & 7 & 8 & 9 & 10 \\
$R_{2}$ & 36 & 49 & 64 & 81 & 100 \\
$\mathrm{R}$ & 35.59 & 48.31 & 62.90 & 79.34 & 97.56 \\
\hline
\end{tabular}

为说明我们的格式是无条件稳定的, 我们分别给出了 $\lambda=10,100,1000,10000$ 的数值结 果, 取网格点 $J-1=100000$. 其中 $\lambda=\tau / h^{2}, \mathrm{CPU} s$ 表示处理机个数, $T_{\text {all }}$ 表示计算 100000 个时间步的总时间, $S_{p}$ 是相对加速比, $E_{f f}$ 表示并行效率. 从表 $2-$ 表 5 可以看出我们所构造

表 2 格式的稳定性和并行性 $(\lambda=10)$

\begin{tabular}{cccccc}
\hline CPUs & 1 & 10 & 20 & 40 & 80 \\
\hline $\max _{j, n}\left|v_{j}^{n}-u_{j}^{n}\right|$ & $1.1244 \mathrm{E}-010$ & $1.1256 \mathrm{E}-010$ & $1.1254 \mathrm{E}-010$ & $1.1256 \mathrm{E}-010$ & $1.1262 \mathrm{E}-010$ \\
$T_{\text {all }}(s)$ & 21802.29 & 2051.44 & 1025.84 & 517.49 & 262.47 \\
$S_{p}$ & 1 & 10.63 & 21.25 & 42.13 & 83.07 \\
$E_{f f}(100 \%)$ & 1 & 1.06 & 1.06 & 1.05 & 1.04 \\
\hline
\end{tabular}


表 3 格式的稳定性和并行性 $(\lambda=100)$

\begin{tabular}{cccccc}
\hline CPUs & 1 & 10 & 20 & 40 & 80 \\
\hline $\max _{j, n}\left|v_{j}^{n}-u_{j}^{n}\right|$ & $1.4152 \mathrm{E}-009$ & $1.3942 \mathrm{E}-009$ & $1.3782 \mathrm{E}-009$ & $1.3404 \mathrm{E}-009$ & $1.2640 \mathrm{E}-009$ \\
$T_{\text {all }}(s)$ & 21927.49 & 2032.37 & 1031.09 & 515.35 & 263.50 \\
$S_{p}$ & 1 & 10.79 & 21.27 & 42.55 & 83.22 \\
$E_{f f}(100 \%)$ & 1 & 1.08 & 1.06 & 1.06 & 1.04 \\
\hline
\end{tabular}

表 4 格式的稳定性和并行性 $(\lambda=1000)$

\begin{tabular}{cccccc}
\hline CPUs & 1 & 10 & 20 & 40 & 80 \\
\hline $\max _{j, n}\left|v_{j}^{n}-u_{j}^{n}\right|$ & $3.8888 \mathrm{E}-008$ & $2.4748 \mathrm{E}-008$ & $1.2339 \mathrm{E}-008$ & $2.0560 \mathrm{E}-008$ & $7.9452 \mathrm{E}-008$ \\
$T_{\text {all }}(s)$ & 22923.82 & 2041.89 & 1025.28 & 511.41 & 262.43 \\
$S_{p}$ & 1 & 11.23 & 22.36 & 44.82 & 87.35 \\
$E_{f f}(100 \%)$ & 1 & 1.12 & 1.12 & 1.12 & 1.09 \\
\hline
\end{tabular}

表 5 格式的稳定性和并行性 $(\lambda=10000)$

\begin{tabular}{cccccc}
\hline CPUs & 1 & 10 & 20 & 40 & 80 \\
\hline $\max _{j, n}\left|v_{j}^{n}-u_{j}^{n}\right|$ & $1.2121 \mathrm{E}-006$ & $2.0323 \mathrm{E}-006$ & $4.9454 \mathrm{E}-006$ & $1.0927 \mathrm{E}-005$ & $2.2914 \mathrm{E}-005$ \\
$T_{\text {all }}(s)$ & 22123.62 & 2076.82 & 1024.24 & 514.36 & 267.47 \\
$S_{p}$ & 1 & 10.65 & 21.60 & 43.01 & 82.71 \\
$E_{f f}(100 \%)$ & 1 & 1.07 & 1.08 & 1.08 & 1.03 \\
\hline
\end{tabular}

的格式是无条件稳定的, 且具有超线性加速比.

\section{8 具有界面外推的并行格式的若干例子及拟开展的研究工作}

8.1 通过在一般格式 (2.4) 中取定参数可得到若干具体的并行格式. 将在并行机上应用这些 格式求解大规模科学与工程计算中的抛物型方程组的定解问题.

对二阶导数 $v_{x x}$ 取 4 类差分逼近: (i) 中心 (隐) 格式 $\mathcal{C}$

(ii) 右格式 $\mathcal{R}$

$$
\frac{v_{j+1}^{n+1}-2 v_{j}^{n+1}+v_{j-1}^{n+1}}{h^{2}}
$$

$$
\frac{\left(2 v_{j+1}^{n}-v_{j+1}^{n-1}\right)-2 v_{j}^{n+1}+v_{j-1}^{n+1}}{h^{2}}
$$

(iii) 左格式 $\mathcal{L}$

$$
\frac{v_{j+1}^{n+1}-2 v_{j}^{n+1}+\left(2 v_{j-1}^{n}-v_{j-1}^{n-1}\right)}{h^{2}}
$$

(iv) Jacobi 型显格式 $\mathcal{J}$

$$
\frac{\left(2 v_{j+1}^{n}-v_{j+1}^{n-1}\right)-2 v_{j}^{n+1}+\left(2 v_{j-1}^{n}-v_{j-1}^{n-1}\right)}{h^{2}} .
$$

一般地, 对每一子区域的内点 $\left\{x_{j}\right\}$ 有 $0<\lambda_{j} \leqslant 1$ 和 $0<\mu_{j} \leqslant 1$. 通常取 $\lambda_{j}=1$ 和 $\mu_{j}=1$, 即格式 $\mathcal{C}$. 于是, 一个内部子区域段可表示为

$$
\mathcal{L C C} \ldots \mathcal{C C R} \text {. }
$$


令 $j_{0}$ 为一固定整数, 满足 $1<j_{0}<J-1$. 令 $x=x_{j_{0}}$ 为区域分解中子区域的内边界点.

例 1 如果有 $\lambda_{j_{0}}=0$, 那么, 在第 $(n+1)$ 层上对 $\left\{v_{j}^{n+1}: j \leqslant j_{0}\right\}$ 的计算与 $\left\{v_{j}^{n+1}: j \geqslant\right.$ $\left.j_{0}+1\right\}$ 无关. 在 $x_{j_{0}}$ 附近格式可表示为

$$
\cdots \mathcal{C C C R C C C} \cdots \text {. }
$$

类似地, 如果有 $\mu_{j_{0}+1}^{n}=0$, 那么, 在第 $(n+1)$ 层上 $\left\{v_{j}^{n+1}: j \geqslant j_{0}+1\right\}$ 的计算与 $\left\{v_{j}^{n+1}: j \leqslant j_{0}\right\}$ 无关. 在 $x_{j_{0}}$ 附近的格式可表示为

$$
\cdots \operatorname{CCC} \mathcal{L C C C} \cdots \text {. }
$$

例 2 如果对一个整数 $n(1 \leqslant n \leqslant N)$, 有 $\lambda_{j_{0}}=0$ 和 $\mu_{j_{0}+1}=0$, 那么, 在第 $(n+1)$ 层上 $\left\{v_{j}^{n+1}: j \leqslant j_{0}\right\}$ 和 $\left\{v_{j}^{n+1}: j \geqslant j_{0}+1\right\}$ 可同时并行求解. 在 $x_{j_{0}}$ 附近的格式可表示为

$$
\cdots \mathcal{C C R} \mathcal{L C C} \cdots,
$$

该格式设计简单, 易于在大规模并行机上实施, 但不满足 $\lambda_{j}+\mu_{j+1} \geqslant 1$.

例 3 如果有 $\lambda_{j_{0}-1}=0, \mu_{j_{0}+1}=0$ 且 $\lambda_{j_{0}}=\mu_{j_{0}}=1$, 那么, 在 $(n+1)$ 层上, 首先对 $\left\{v_{j}^{n+1}: j \leqslant j_{0}-1\right\}$ 和 $\left\{v_{j}^{n+1}: j \geqslant j_{0}+1\right\}$ 同时并行求解; 然后, 由已得到的 $v_{j_{0}-1}^{n+1}$ 和 $v_{j_{0}+1}^{n+1}$, 可 用隐式格式计算 $v_{j_{0}}^{n+1}$. 在 $x_{j_{0}}$ 附近的格式可表示为

$$
\text { ‥ CCRCLCCC.. }
$$

该格式设计简单, 易于在大规模并行机上实施, 且满足 $\lambda_{j}+\mu_{j+1} \geqslant 1$.

例 4 如果有 $\lambda_{j_{0}}=\mu_{j_{0}}=0$, 且其余的 $\lambda_{j}=\mu_{j}=1$, 那么, 在 $(n+1)$ 层上, 首先求出 $v_{j_{0}}^{n+1}$, 然后, 利用已得到的 $v_{j_{0}}^{n+1}$ 对 $\left\{v_{j}^{n+1}: j \leqslant j_{0}-1\right\}$ 和 $\left\{u_{j}^{n+1}: j \geqslant j_{0}+1\right\}$ 可用隐式格式同 时并行求解. 在 $x_{j_{0}}$ 附近的格式可表示为

$$
\cdots \mathcal{C C C} \mathcal{J C C C} \cdots,
$$

该格式设计简单, 易于在大规模并行机上实施, 且满足 $\lambda_{j}+\mu_{j+1} \geqslant 1$.

8.2 可将本文中的格式的构造及证明结果推广到多维非线性抛物型方程和散度型抛物方程 的定解问题等.

特别重要的是, 我们将研究并行格式的守恒性问题. 除文献 [12] 中提出的子隐式格式外, 其余的并行格式的设计未专门考虑格式的守恒性. 一般说来, 原来的隐式格式总是守恒格式, 在每个子区域内部使用原来的隐式格式, 在子区域内界面处采用与之不一致的具有某种显式 性质的格式, 这会带来守恒性的损失, 因此, 必须考虑越过子区域内界面处的守恒性 (流的连 续性). 即我们需要在现有并行格式的基础上, 在子区域内界面处进行保证守恒性的隐式修正. 例如, 对于上述例 4 给出的并行格式, 子区域内界面 $x_{k}$ 处先取 Jacobi 型显格式 $\mathcal{J}$ 进行界 面处的预估, 记所得预估值为 $\bar{v}_{k}^{n+1}$, 在子区域内部用隐式格式同时并行求解后得到 $v_{k+1}^{n+1}$ 和 $v_{k-1}^{n+1}$, 最后采用如下格式进行守恒修正, 得到 $v_{k}^{n+1}$ :

$$
\frac{v_{k}^{n+1}-v_{k}^{n}}{\tau}=\frac{1}{h^{2}}\left(v_{k+1}^{n+1}-\bar{v}_{k}^{n+1}-\left(\bar{v}_{k}^{n+1}-v_{k-1}^{n+1}\right)\right) .
$$

在以后的论文中将进一步深入讨论守恒的并行格式的设计及其性质分析.

\section{参考文献}

1 Evans D J. Alternating Group Explicit method for the diffusion equations. Appl Math Modeling, 1985, 19: $201-206$ 
2 Zhang B L, Li W Z. On alternating segment Crank-Nicolson scheme. Parallel Computing, 1994, 20: 897-902

3 Han Z, Fu H Y, Shen L J. Pure alternating segment Explicit-Implicit method for the diffusion equations. International Journal of Computer Mathematics, 1993, 51: 8-15

4 Yuan G W, Shen L J, Zhou Y L. Unconditional stability of parallel alternating difference schemes for semilinear parabolic systems. Applied Mathematics and Computation, 2001, 117: 267-283

5 Yuan G W, Zuo F L. Parallel differences schemes for heat conduction equation. International Journal of Computer Mathematics, 2003, 80: 995-999

6 Dawson C N, Du Q, Dupont T F. A finite difference domain decomposition algorithm for numerical solution of the heat equation. Mathematics of Computation, 1991, 57(195): 63-71

7 Dawson C N, Dupont T F. Explicit/implicit conservative domain decomposition procedures for parabolic problems based on block-centered finite differences. SIAM J Numer Anal, 1994, 31: 1045-1061

8. Du Q, Mu M, Wu Z N. Efficient parallel algorithms for parabolic problems. SIAM J Numer Anal, 2001, 39(5): 1469-1487

9 Yuan G W, Shen L J, Zhou Y L. Parallel differences schemes for parabolic problem. In: Proceeding of 2002 5th International Conference on Algorithms and Architectures for Parallel Processing. IEEE Computer Society, 2002, 238-242

10 袁光伟, 杭旭登. 热传导方程基于界面修正的迭代并行计算方法. 数值计算与计算机应用, 2006, 27(1): $67-80$

11 Zhou Y L. Applications of Discrete Functional Analysis to Finite Difference Method. Beijing: International Academic Publishers, 1990

12 Eltgroth P G, Seager M K. The sub-implicit method: New multiprocessor algorithms for old implicit codes. Parallel Computing, 1988, 8: 155-163 